\title{
Die Übertrittsempfehlung - zufällig oder zuverlässig? \\ Analyse der Determinanten und Konstanz von Lehrerempfehlungen bei Schulüber- tritten während sieben Jahren
}

\section{Franz Baeriswyl, Christian Wandeler und Oliver Christ}

Übergänge sind ein zentrales Merkmal des mitteleuropäischen Schulsystems und sind wichtige Phasen für das Kind. Bei gegliederten Schulsystemen wird der Eintritt in die Sekundarstufe I besonders bedeutsam, weil mit der Zuweisung zu einem der Schultypen implizit auch die Zuteilung von Bildungschancen verbunden ist. Für die Zuweisung ist die Lehrerempfehlung ein weitverbreitetes Kriterium. Dieser Beitrag untersucht, ob sich die Determinanten der Lehrerempfehlung in sieben aufeinanderfolgenden Jahrgängen unterscheiden und sich ein stabiles Modell der Lehrerempfehlung erstellen lässt.

Die Daten stammen von 5332 Schülerinnen und Schülern des Übertrittsverfahrens von der Grundschule in die Sekundarschule I der deutschsprachigen Schulen des Kantons Freiburg (Schweiz). Unter Berücksichtigung der Schachtelung der Daten auf Klassenebene wurde für sieben verschiedenen Jahre das gleiche Modell spezifiziert. Die Modellierung der Lehrerempfehlung erwies sich über die verschiedenen Jahrgänge hinweg als statistisch nicht signifikant unterschiedlich, jedoch existieren teilweise unterschiedliche Tendenzen der Gewichtung von Variablen. Lehrpersonen achten stärker auf Leistungsstand-als auf Leistungsprozessbeurteilungen, wobei Einschätzungen des Leistungsstandes in Mathematik und Deutsch und der kognitiven Fähigkeiten die am stärksten gewichteten Prädiktoren sind.

\section{Einleitung}

Der Übergang von der Primar- oder Grundschule in die Sekundarschule ist für das betroffene Individuum, seine Familie, die Primarlehrperson, die abnehmende Schule und das weiterführende Berufs- und Schulbildungssystem von besonderer Bedeutung. Für die Schülerin und den Schüler als Individuum verursacht er psychischen Stress, der jedoch nach dem Schulwechsel abklingt (Elben, Lohaus, Ball \& Klein-Hessling, 2003), aber dennoch grosse psychische Anpassungen verlangt (Ball, Lohaus \& Miebach, 2006), die je nach Schulform unterschiedlich ausfallen (Valtin \& Wagner, 2004). Die Familie, insbesondere die El- 
tern entwickeln Bildungsaspirationen (Bourdieu, 1983; Ditton, 2007; Neuenschwander et al., 2005), die für die Zuteilung unterschiedlich berücksichtigt werden. In Deutschland entscheiden in der Mehrzahl der Länder die Eltern über den Besuch der Sekundarschulform ihres Kindes (Arnold, Bos, Richert \& Stubbe, 2007, S. 272). Das Berufs- und Schulbildungssystem ist insofern betroffen, als in der Schweiz, Deutschland und Österreich eine hierarchische Gliederung der Sekundarstufe I vorherrscht (Maaz, Neumann \& Trautwein, in Druck), was Übertrittsentscheiden eine besondere Bedeutung verleiht, weil einmal gemachte Zuweisungen nur schwer abänderbar sind. Dieser, bereits von Fend (1981) beschriebene «Halteeffekt» kann mit einer Gesamtschulstruktur oder mit einer institutionellen Öffnung des Bildungssystems bis zur beruflichen Grundbildung und einer offeneren Zugangsregelung für höhere Ausbildungen/Weiterbildungen entschärft werden (Trautwein, Baeriswyl, Lüdcke \& Wandeler, in Druck). Die Öffnung des Bildungssystems bei gleichzeitig früher Selektion entlastet die Entscheidungsträger angesichts des nachgewiesenen Schereneffektes (Becker, Lüdtke, Trautwein \& Baumert, 2006) nicht von einer grossen Verantwortung. Die Ausbildung in den hierarchisch gegliederten Abteilungen der Sekundarstufe I stellen nach Baumert (Baumert, Stanat \& Watermann 2006; Becker et al., 2006; Neumann et al., 2007; Trautwein et al., in Druck) «differentielle Entwicklungsmilieus» dar. Bei vergleichbaren Leistungsfähigkeiten zeigen Schülerinnen und Schüler einer anspruchsvolleren Sekundarabteilung bereits nach einem Jahr grössere Leistungssteigerungen. In einer Abteilung mit tieferen Ansprüchen können sich Leistungsfähigkeiten gar rückentwickeln (Becker et al, 2006; Gruehn, 2000). Fehlentscheidungen bei Abteilungszuweisungen werden später nur selten korrigiert (Baumert, Trautwein \& Artelt, 2003, S. 310). Demnach ist der Prognosequalität der Zuweisungsentscheidung besondere Beachtung zu schenken. Auch Neuenschwander et al. (2007, S. 83) berichten eine geringe Durchlässigkeit zwischen den Abteilungen der Sekundarstufe I und weisen auf die Bedeutung einer optimalen Passung zwischen individueller Leistungsfähigkeit bei positivem Selbstwert und der institutionellen Erwartungserfüllung hin. Griebel (2004) bezeichnet Übergänge als ein «dynamisches, prozesshaftes Geschehen über längere Zeit, in dem es einerseits zu einer Verdichtung unterschiedlicher Belastungsfaktoren kommt, das andererseits aber auch Chancen für Lernprozesse eröffnet» (S. 41).

\section{Theoretischer Hintergrund}

Die Untersuchungen zur Validität von Schulnoten aus den 60er und 70er Jahren zeigen eine geradezu desaströse Situation (Ingenkamp, 1976; Ziegenspeck, 1973). Die einzelne Prüfungsnote drückt höchstens eine Rangreihe der Klassenmitglieder aus; auf jeden Fall ist die Übereinstimmung einer Prüfungsnote mit der individuellen Leistungsfähigkeit gering. Trotz der seit wenigstens vierzig Jah- 
ren nachgewiesenen mangelhaften Reliabilität und Validität der Schulnoten, konnten diese nicht durch adäquatere Beurteilungsformen ersetzt werden. In der Schweiz wurden während der letzten zwanzig Jahren teilweise Vergleichsprüfungen und Tests bei Übertrittsverfahren in die Sekundarstufe I eliminiert und die Lehrerempfehlung zum zentralen Zuweisungselement gemacht (Vögeli-Mantovani, 1999). In Deutschland setzte eine ähnliche Entwicklung schon früher ein. Viele Länder haben bereits das Schulwahlrecht der Eltern der Lehrerempfehlung vorangestellt.

Jürgens (1989) plädierte dafür, dass das Elternwahlrecht der Schulabteilung erhalten bleibt, weil viele für eine Abteilung als «ungeeignet» empfohlene Kinder dank der Elternempfehlung gleichwohl erfolgreich waren. Trotzdem wird in den letzten Jahren die Lehrerempfehlung wieder aufgewertet. Wobei in letzter Zeit wiederum eine Tendenz zur Rücknahme der alleinigen Elternentscheidung zu Gunsten einer höheren Gewichtung der Schulformempfehlung der Lehrkraft auszumachen ist (Arnold et al., 2007, S. 292; Ditton, 2007; Thiel, 2006). Dies wohl deshalb, weil der Zuweisungsentscheid der Eltern zunehmend von ihren Bildungsambitionen und ihrem sozioökonomischeen Status und weniger vom schulischen Leistungsvermögen ihres Kindes geprägt ist (Baumert \& Schümer, 2001; Baumert, Trautwein \& Artelt, 2003; Baeriswyl, Wandeler, Trautwein \& Oswald; 2006 Ditton \& Krüsken, 2006; Ditton, Krüsken \& Schauenberg, 2005) und so den Anspruch eines sozialgerechten Bildungssystems unterläuft. Arnold et al. (2007, S. 277) berichteten, dass die Übereinstimmung der Lehrerempfehlung mit der Elternempfehlung zur Schulwahl von 2001 bis 2006 beachtlich abgenommen habe und schreiben dies den immer anspruchsvolleren Bildungsaspirationen der Eltern zu.

Die Lehrerempfehlung (Übertrittsempfehlung der Primarlehrperson) beim Übertritt von der Primar- in die Sekundarschule wurde bereits gut erforscht (Bos et al., 2004; Ditton \& Krüsken, 2006; Jürgens, 1989; Ophuysen van, 2006; Stahl, 2007; Stubbe \& Bos, 2008; Thiel, 2006). Diese Autoren haben die Lehrerempfehlung mit unterschiedlichen Verfahren modelliert. Die Ergebnisse zeigen in hoher Übereinstimmung, dass die Schulnote in Deutsch und Mathematik den grössten Teil der Varianz der Lehrerempfehlung aufklärt. Schulnoten bilden jedoch nicht objektiv Leistungsfähigkeiten ab, sondern unterliegen Attributions-, Wahrnehmungseffekten und weisen eine relativ geringe Reliabilität auf. Trotzdem bilden sie die wichtigsten Determinanten der Lehrerempfehlung. Für eine Prognose des Schulerfolges in der Sekundarstufe I ist beim Übertrittsverfahren die Testleistung vor der Schulnote der beste Prädiktor und die Lehrerempfehlung verliert ihre Vorhersagekraft (Baeriswyl, Trautwein \& Wandeler, 2008). Thiel (2006) kam in seiner Dissertation zur Modellierung der Bildungsgangempfehlung zum Schluss, dass sie «... im wesentlichen mit den Schulleistungen» zusammenhängen, wie sie sich in Schulnoten und Leistungsergebnissen manifestieren - «Andere Schülermerkmale haben keinen direkten Einfluss» (Thiel, 2006, S. 247). 
Stahl (2007, S. 192) liess von Lehrpersonen die Wichtigkeit der bei der Übertrittsempfehlung berücksichtigten Faktoren einschätzen. Dabei wurden schulische Leistungen, Anstrengungsbereitschaft und Begabung als «sehr wichtig» betrachtet. Als "wichtig» wurde die Unterstützung durch die Eltern, die deutsche Sprache und das Sozialverhalten genannt. Sie stellte zudem fest, dass einer Lehrerempfehlung je nach Schulabteilung spezifische Entscheidungsprozesse zugrunde liegen. So interagierte die Abteilungsempfehlung mit der Bewertung schulischer Leistung: für Hauptschüler bestand ein enger Zusammenhang zwischen schwacher Schulleistung und negativer Bewertung der Begabung, was nicht für Gymnasialempfehlungen zutraf; eine positive Leistungsbewertung ging jedoch nicht unbedingt mit hoher Begabung einher. Ebenso war ein positives Sozialverhalten für die Übertrittsempfehlung der Mädchen weniger wichtig als für jene der Knaben. Ein positiv bewertetes Sozialverhalten wurde bei den Knaben eher als Grund für eine Gymnasialempfehlung angeführt und ein negativ bewertetes eher als Rechtfertigung für eine Hauptschulempfehlung eingesetzt. Wurden Lehrpersonen nach ihren Entscheidungsmerkmalen für die Lehrerempfehlung befragt, hoben sie die Orientierung an der Leistung hervor und gaben an, ihre Entscheidung nicht an Herkunft und Sozialstatus auszurichten (Stahl, 2007, S. 194). Stahl (2007), wie u. a. auch Bos, Voss et al. (2004), Ditton, Krüsken und Schauenberg (2005), stellten jedoch fest, dass Kinder mit einem tieferen sozioökonomischen Status um vergleichbare Leistungsbewertungen zu erzielen wesentlich höhere Leistungen erbringen müssen als sozial besser gestellte. Demnach sind neben den Leistungsmerkmalen schulische Kontextmerkmale wie Wohnregion, sozioökonomischer Hintergrund, Migrationsstatus, und die Klassenzusammensetzung bezüglich dieser Merkmale zu berücksichtigen (Ditton, 2007; Thiel, 2006). Je nach Wohnregion variiert der für das Gymnasium empfohlene Prozentsatz erheblich (Thiel, 2006).

Bos, Voss et al. (2004) modellierten im Rahmen des IGLU Projektes ein Prognosemodell der Schullaufbahnempfehlung der Lehrpersonen, das ebenfalls Kontextmerkmale berücksichtigte. Darin wurden Leistungsmerkmale (Testleistungen in Mathematik und Lesen, sowie für kognitive Fähigkeiten), schulnahe Kriterien (Deutsch-, Mathematiknote, Anstrengungsbereitschaft, sowie Leistungsangst), und soziale Merkmale (Geschlecht, Migrationsstatus und sozioökonimischer Hintergrund) unterschieden. Dieses Modell klärte für fünf Bundesländer insgesamt $68 \%$ der Variabilität der Lehrerempfehlung auf. Für alle untersuchten Bundesländer zeigte das Regressionsmodell, dass die Deutschnote, gefolgt von der Mathematiknote die grösste Erklärungskraft hat. Weiter erwies sich einzig noch der sozioökonomische Status der Eltern bei allen Bundesländern als signifikant prädiktiv. Stubbe und Bos (2008) überprüften dieses Modell pfadanalytisch. Mit der Deutsch- und Mathematiknote konnten sie 77\% (bei Bos et al. 2004 waren es 66\%) der Lehrerempfehlung aufklären. Ihr Gesamtmodell mit zehn Prädiktoren erklärte die Variabilität der Lehrerempfehlung zu 79\%, dabei trugen die Deutsch- und Mathematiknoten zusammengefasst mit 
$52 \%$ am meisten zur Aufklärung bei. Mit Ausnahme des Schülergeschlechts und des Migrationsstatus wirken alle Hintergrundvariablen (wie kognitive Fähigkeiten, Anstrengungsbereitschaft, Prüfungsangst, ISEI und die Testleistung in Lesen und Mathematik) sowohl mediiert über die Deutsch- und Mathematiknote wie auch direkt auf die Lehrerempfehlung. Stahl (2007) modellierte die Lehrerempfehlung mit einem Strukturgleichungsmodell und berücksichtigte die Lehrereinschätzungen der Begabung, die Einschätzung des Elternhauses (förderliche Rahmenbedingungen, die Unterstützung durch das Elternhaus) und der sprachlichen Ausdrucksfähigkeit als vermittelnde Variablen auf die Testleistung, die Noten und die Lehrerempfehlung. Sie untersuchte hiermit mögliche Einflussfaktoren auf die Lehrereinschätzung von Schülermerkmalen und stellt fest, dass für die Lehrerempfehlung neben den Noten, die eingeschätzte sprachliche Ausdrucksfähigkeit und die Begabung relevant sind (Stahl, 2007, S. 191). Der sozioökonomische Hintergrund eines Schülers wirkte indirekt über die Testleistung und die Lehrereinschätzungen auf das Lehrerurteil. Mit dem Gesamtmodell konnte Stahl (2007) 70\% der Varianz der Lehrerempfehlung aufklären und $82 \%$ der Schulnoten. Thiel (2006) modellierte die Bildungsgangempfehlung der Lehrperson mit verschiedenen Methoden. Pfadanalytisch konnten $82 \%$ ihrer Varianz aufgeklärt werden, wobei die Schulnoten den grössten direkten Einfluss aufwiesen. Ditton, Krüsken und Schauenberg (2005) konnten 76\% der Lehrerempfehlung für die gymnasiale Schulform und 79\% einer Hauptschul-Empfehlung aufklären. In der vorliegenden Arbeit wird im Sinne einer Weiterführung der Ausdifferenzierung des Konstrukts «Lehrerempfehlung» ihre zeitliche Stabilität untersucht. Werden für ihre Bildung jedes Jahr ungefähr dieselben Variablen in vergleichbarem Ausmass berücksichtigt?

Diese neuesten Untersuchungen zeigen die Übertrittsempfehlung einer Lehrperson als komplexes Konstrukt, das mehrheitlich durch die Noten, die Testleistung (sofern sie vorliegt) und Wahrnehmungsfaktoren der Lehrperson über die Schülerpersönlichkeit (wie z.B. Begabungseinschätzung) bestimmt wird (Stahl, 2007).

Mit der Übertritts- oder Zuweisungsempfehlung gibt die Lehrperson implizit auch eine Prognose ab. Ein grundlegendes Prognosemodell für den pädagogisch-psychologischen Bereich hat Krapp (1979) entwickelt. Thiel (2006, S. 43) meinte dazu: «Eine Prognose ist nur dann sinnvoll möglich, wenn angenommen werden darf, dass die Parameter des Prognosemodells unverändert bleiben, d.h. konstant sind.» Diese Konstanzannahmen bedingen denn auch die Gültigkeit der Prognose (Sauer \& Gamsjäger, 1996). Ihnen Genüge zu tun ist im pädagogischen Bereich wesentlich schwieriger als beispielsweise in der Physik, weshalb sie im Zusammenhang mit der Lehrerempfehlung selten thematisiert wird. 


\section{Das Deutschfreiburger übertrittsverfahren}

Im Mittelpunkt des vorliegenden Beitrags steht das Deutschfreiburger Übertrittsmodell, das in den 1990er Jahren entwickelt wurde, um das Übertrittsverfahren zu standardisieren, hohe Transparenz zu erzeugen, Chancengleichheit zu gewährleisten und eine Revidierbarkeit der eingeschlagenen Bildungswege zu ermöglichen (Baeriswyl et al., 2006). Der deutschsprachige Teil des Kantons Freiburg führt die Sekundarstufe I in Form einer Orientierungsschule mit drei Abteilungen. Die Progymnasialabteilung (höhere Anforderungen), die Sekundarabteilung (mittlere Anforderungen) und die Realabteilung (Grundanforderungen) befinden sich im gleichen Gebäude und pflegen teilweise gemeinsame Aktivitäten (z.B. Turnunterricht, Sport- oder Projektwochen). Nach der obligatorischen Schulzeit von neun Jahren sind Eintritte ins Gymnasium oder in eine Berufsausbildung möglich. Bei genügenden Schulleistungen (Notendurchschnitt der Hauptfächer 4.0 bei der Notenskala von $6=$ sehr gut, 5 = gut, 4 = genügend, 3 = ungenügend) ist der Übertritt von der progymnasialen Abteilung ins Gymnasium prüfungsfrei. Der Übertritt von der Sekundarabteilung ins Gymnasium ist ebenfalls prüfungsfrei, wenn in den Hauptfächern (Deutsch, Mathematik, Naturkunde/Realien (naturwissenschaftliche Fächer) und Französisch) ein Notendurchschnitt von 5.0 (gut) erreicht wird. Bei Nichterreichen dieses Notendurchschnitts kann für den Zugang zum Gymnasium eine Aufnahmeprüfung abgelegt werden. Unter denselben Bedingungen sind Eintritte in die Berufsmaturitätsausbildung möglich. Für die Schülerinnen und Schüler der Realabteilung sind keine prüfungsfreien Übertritte in diese weiterführenden Schulen möglich und Übergänge in die Berufsausbildung am häufigsten. Hier entwickeln die einzelnen Berufsverbände oft eigene Aufnahmeverfahren.

In der vorliegenden Studie wird der Übertritt von der 6. Klasse der Primarschule ins 7. Schuljahr der Sekundarstufe I untersucht. Das Übertrittsverfahren beruht auf einem dynamischen Entscheidungsmodell, das auf qualitativer Datenanalyse und direkter Kommunikation der Entscheidungsbeteiligten beruht. Es umfasst drei Hauptelemente: Die Übertrittsempfehlung der Lehrperson, die Übertrittsempfehlung der Eltern, sowie die Leistungen der Schülerinnen und Schüler in einer standardisierten Vergleichsprüfung. Die Primarlehrperson dokumentiert ihre Empfehlung mit drei Elementen. Zum einen beurteilt sie jeweils im Zeugnis des ersten Semesters eines Schuljahres die Lernprozesse oder Lernfortschritte (kommt bei der Erarbeitung der Lernziele gut voran; hat bei der Erarbeitung der Lernziele Schwierigkeiten; hat ... viele Schwierigkeiten). Diese Lernprozessbeurteilung soll vorwiegend die Lernfortschritte, bezogen auf den Lehrplan, bewerten. Zum anderen führt sie ein Beobachtungs- und Beurteilungsinstrument mit Beurteilungskriterien zu kognitiven Fähigkeiten und zum Lern- und Arbeitsverhalten. Dieses Beurteilungsinstrument soll gewährleisten, dass auch temporäres Underachievement bzw. besonderer Fleiss für die Übergangsentscheidungen mitberücksichtigt werden können. Als drittes Element 
gibt die Lehrperson unmittelbar vor der Vergleichsprüfung pro Fach eine Lernstandsbeurteilung ab (hat die Lernziele sehr gut erreicht; ... gut erreicht; ... erreicht; ... nicht erreicht). Die Lernstandsbeurteilung soll von Lehrpersonen und Eltern als kriteriumsorientierte Bewertung verstanden werden. Auf der Basis von Beurteilungsbogen und der Prozess- und Lernstandsbeurteilung formuliert die Lehrperson eine Empfehlung für die Zuweisung zu einer der drei Abteilungen. Dabei kann sie die Empfehlung differenzieren mit Abteilung XY empfoblen oder Abteilung XY bedingt empfohlen.

Das zweite Hauptelement ist die Übertrittsempfehlung der Eltern, die aus einem direkten Kommunikationsprozess mit der Lehrperson hervor geht. Die Lehrperson muss die Eltern vor der Übertrittsprüfung zu einem Gespräch einladen, in dem sie ihnen die Lehrerempfehlung und die Beurteilung offen legt. Nach diesem Gespräch erhalten die Eltern ein Formular, auf dem sie ihre Empfehlung schriftlich festhalten und der Lehrperson zurücksenden. Den Eltern ist es freigestellt, bei ihrer Empfehlung von der Empfehlung der Lehrperson abzuweichen. Die Übertrittsempfehlungen von Lehrperson und Eltern, die Beobachtungsbögen und die Zeugnisbeurteilungen werden zentral festgehalten.

Als drittes Hauptelement folgt eine standardisiert durchgeführte Vergleichsprüfung für alle Schülerinnen und Schüler des Kantons in den Bereichen Deutsch und Mathematik. Der Leistungstest wird jedes Jahr auf Grund des kantonalen Curriculums für die 5. und den Anfang der 6. Klasse konzipiert und in einem anderen Kanton, welcher ein vergleichbares Curriculum hat, auf Verständlichkeit, Aufgabenschwierigkeit erprobt. Er wird von allen Schülerinnen und Schülern am selben Tag bearbeitet (in den ausgewerteten Jahren jeweils in der ersten Hälfte des Monats März). Die Auswertung erfolgt durch Lehrkräfte aus dem Sekundarschulbereich. Für jede Schülerin und jeden Schüler wird das Gesamtprüfungsergebnis als Prozentrang festgehalten und mit der Lehrerempfehlung verglichen. Durch dieses Vorgehen fungiert der jeweilige Jahrgang als soziale Referenznorm.

Der Zuweisungsprozess läuft in mehreren Phasen ab. In der ersten Phase wird zuerst die Übereinstimmung zwischen Lehrerempfehlung mit dem Prüfungsergebnis überprüft. Übereinstimmung herrscht dann, wenn die Lehrerempfehlung für eine Abteilung mit erweiterten Ansprüchen (z.B. Progymnasium empfohlen) mit einem hohen Prozentrang beim Prüfungsergebnis (PR > 85) einhergeht oder eine Empfehlung für die Realabteilung mit einem tiefen Prüfungsergebnis $(\mathrm{PR}<$ 30). Bei Übereinstimmung zwischen Lehrerempfehlung und Prüfungsleistung folgt eine direkte Zuweisung zu der von der Lehrperson empfohlenen Abteilung. Bei Diskrepanz zwischen Lehrerempfehlung und Prüfungsergebnis wird der Zuweisungsentscheid als offen bezeichnet (z. B. Lehrerempfehlung Realabteilung und Prüfungsergebnis PR > 50).

Lautet der erste Zuweisungsentscheid offen folgt die zweite Phase, in der die zuweisungsberechtigte Schuldirektorin oder der Schuldirektor der Sekundarstufe I gemeinsam mit der Primarlehrperson und den Eltern auf der Basis von 
Beurteilungs- und Prüfungsdokumenten einen Zuweisungsentscheid treffen.

Die dritte Phase ist das erste Semester in der Orientierungsschule (7. Schuljahr). Während dieser Zeit kann zu jedem Zeitpunkt, auf Antrag der Eltern oder der Lehrpersonen der Sekundarstufe I, ein Abteilungswechsel vorgenommen werden - in dieser Phase herrscht also zumindest theoretisch eine grosse Systemdurchlässigkeit. Das Deutschfreiburger Übertrittsmodell (siehe Baeriswyl et al., 2006) vereinigt viele der Merkmale, die im Hinblick auf die Zuverlässigkeit eines Zuweisungsverfahrens in neuerer Literatur gefordert wurden (Bos et al., 2003; Ingenkamp \& Lissmann, 2005).

Beim Deutschfreiburger Übertrittsverfahren gibt es bildungspolitische Rahmenvorgaben bezüglich der Abteilungsanteile eines Jahrganges. Danach sollten der Progymnasialabteilung 15 bis $20 \%$, der allgemeinen Sekundarabteilung 45 bis $55 \%$ und der Realabteilung 30 bis 35\% der Jahrgangsklasse zugeteilt werden. Weil die Primarlehrpersonen diesbezüglich auch informiert werden, dass diese Vorgaben nicht für jede einzelne Klasse, sondern für den gesamten Kantonsteil gelten soll, werden sie auch nicht strikte befolgt, zumal kaum mehr als $25 \%$ der Realabteilung und eher 25 bis $30 \%$ der Progymnasialabteilung zugewiesen werden.

\section{Fragestellungen}

\section{Überprüfung der zeitlichen Stabilität der Genese von Lehrerempfehlungen}

In der ersten Fragestellung wird erstmals die zeitliche Stabilität der Genese von Lehrerempfehlungen bei Übertrittsverfahren untersucht. Bislang liegen unserem Wissen nach keine publizierten Untersuchungen zu dieser Thematik vor. Dass die Lehrerempfehlungen über die Jahre hinweg konstant nach denselben Kriterien gebildet werden, ist eine Frage der Gerechtigkeit der Zuweisung und somit ein bildungspolitisch relevantes Anliegen.

Bos, Voss et al. (2004) erhielten für verschiedene Bundesländer unterschiedliche Prädiktionsmodelle der Übergangsempfehlung. Diese Unterschiede zwischen den Bundesländern sind bezüglich der Zuweisungsgerechtigkeit weniger störend als die zeitlichen Veränderungen innerhalb einer Region, weil regional die Quoten der Abteilungen ebenfalls grosse Unterschiede aufwiesen und die Schulsysteme auf unterschiedliche Abteilungsquoten ausgerichtet sind. Die $\mathrm{Zu}-$ weisungsgerechtigkeit leidet hingegen stärker, wenn sich in derselben Region die Einflussgrössen auf die Lehrerempfehlung von Jahr zu Jahr stark unterscheiden. Weil jedoch keine Richtlinien zur Bildung der Lehrerempfehlung vorliegen, und jedes Jahr andere Lehrergruppen die 6. Klassen unterrichten, ist davon auszugehen, dass die Kriterien jährlich anders gewichtet werden. Ebenso kann wegen des Fehlens von längsschnittlichen Forschungsergebnissen nicht ausgeschlossen werden, dass das Konstrukt "Lehrerempfehlung», das stark von der individuellen 
Lehrerwahrnehmung geprägt ist (vgl. Stahl, 2007) keine zeitliche Stabilität aufweist. Dies hätte allerdings negative Auswirkungen auf die Bildungsgerechtigkeit, weil Kinder mit vergleichbaren Leistungsfähigkeiten je nach Jahr einer anderen Abteilung zugewiesen würden. Dies aus dem Grund, weil die Lehrerempfehlung implizit als zuverlässiger eingestuft wird als die punktuelle Prüfung und sie beim definitiven Zuweisungsentscheid tendenziell stärker gewichtet wird als das Prüfungsergebnis (Baeriswyl, Trautwein \& Wandeler, 2008).

\section{Einfluss von Beurteilungsformen auf die Lehrerempfehlung}

Die zweite Fragestellung bezieht sich auf die Beurteilungsform im Unterricht. Das damalige Zeugnis im deutschsprachigen Teil des Kantons Freiburg privilegierte eine eher individualnormorientierte Beurteilung am Ende des ersten Semesters (kommt bei der Erarbeitung der Lernziele gut voran ...; hat bei der Erarbeitung der Lernziele Schwierigkeiten; ... grosse Schwierigkeiten). Der individuelle Fortschritt bezieht sich bei dieser Beurteilung auf die Lernziele ${ }^{1}$. Diese Kombination von Individualnorm und Kriteriumsnorm wurde in der bisherigen Forschung zur Schülerbeurteilung nicht besonders beachtet (Rheinberg, 1982; 2001; Rheinberg \& Krug, 1999; Mischo \& Rheinberg, 1995). Die Untersuchungen von Rheinberg zeigen einen bedeutsamen Zusammenhang zwischen der angewendeten Bezugsnorm und der Motivation und dem Selbstkonzept der Schüler (Rheinberg, 2006). Bei der Lernstandsbeurteilung hat die Lehrperson im Kanton Freiburg den Auftrag, den Leistungsstand, bezogen auf die Lernziele des Lehrplans zu beurteilen. Es handelt sich hier um eine kriteriumsnorm-bezogene Beurteilung (Rheinberg, 2006). Bei der Modellierung der Lehrerempfehlung soll die Wirkung dieser beiden Beurteilungsformen auf das Bilden der Lehrerempfehlung untersucht werden. Es ist davon auszugehen, dass die Lehrperson die Lernstandsbeurteilung als das für die Übertrittsempfehlung bedeutsamere Kriterium betrachtet als die Lernprozessbeurteilung.

Stubbe und Bos, (2008) berichten, dass die Deutsch- und Mathematiknoten die Varianz der Lehrerempfehlung zu 55\% aufklären. Weil sich die Lernstandsbeurteilung auf eine kriteriumsnorm-orientierte Beurteilung fokussiert und nicht auf eine sozialnorm-orientierte (wie Noten), gehen wir davon aus, dass mit der Lernstandsbeurteilung in Deutsch und Mathematik eher mehr Varianz aufgeklärt wird als durch Noten. Diese Fragestellung kann jedoch aufgrund der Daten nicht bearbeitet werden.

\section{Einfluss von fachspezifischen Lernstandsbeurteilungen} Mit der dritten Fragestellung soll der spezifische Einfluss der fachspezifischen Lernstandsbeurteilung auf die Bildung der Lehrerempfehlung überprüft werden. Würden die Lehrpersonen ausgeprägt nach dem Prinzip "teaching to the test» unterrichten, würden sie generell eine sehr hohe Übereinstimmung der Mathematik- und Deutschleistungen mit den Ergebnissen der entsprechenden Vergleichsprüfung anstreben. Wenn dem so wäre, würden die Leistungen in den an- 
deren Fächern (Französisch, Naturkunde) die Bildung der Lehrerempfehlung nur geringfügig oder nicht beeinflussen. Mit dieser Fragestellung soll überprüft werden, welche Fachleistungen die Lehrerempfehlung beeinflussen und wie zeitlich stabil diese Einflüsse sind.

\section{Methode}

\section{Stichprobe}

Die vorliegende Studie ist Teil eines Forschungsprojektes der Abteilung Lehrdiplom Sekundarstufe II der Universität Freiburg/Schweiz, in dem Prozesse und Einflussgrössen bei der Bildung des Übertritts von der Primarstufe in die Sekundarstufe I untersucht werden. In dieser Studie verwenden wir die Daten der Schülerinnen und Schüler, die in den Jahren 1998 bis 2004 nach Abschluss der sechsten Primarschulklasse in die Sekundarstufe I übertraten. Während diesen sieben Jahren wurde das Verfahren nicht verändert. Die Primarschule dauert sechs Jahre und ist ungegliedert. Wenige Schulkreise führten noch eine Kleinklasse, wobei die meisten sämtliche Kinder integrieren und die Schwächsten individuellen Lernzielen folgen und heilpädagogisch in den Primarklassen begleitet werden. Weil nicht sämtliche Kinder aus den Kleinklassen am Übertrittsverfahren teilnehmen, wurden diese und jene, die den Regelklassen mit heilpädagogischen Stützunterricht und speziellen Lernzielen folgten, in dieser Studie nicht berücksichtigt. Ebenfalls aus der Stichprobe ausgeschlossen wurden Kinder, die die Primarschulstufe nicht im Kanton Freiburg besuchten; leistungsschwache Kinder, welche aus pädagogischen und psychologischen Gründen an der Prüfung teilnahmen, um die Prüfung zu erleben wie alle anderen ${ }^{2}$ und das kleinste Orientierungsschulzentrum, das damals keine Progymnasialabteilung führte und die Real- und Sekundarklassen in den Hauptfächern in drei Leistungsgruppen gliederte. Tabelle 1 zeigt die Anzahl Kinder der 6. Primarklasse und jene, die für die vorliegende Studie ausgeschlossen worden sind.

Tabelle 1: Berücksichtigte und ausgeschlossenen Fälle, Geschlechtsverteilung der berücksichtigen Fälle

\begin{tabular}{|cccccc|}
\hline Jahr & $\begin{array}{c}\text { Anzahl im } \\
\text { Übertritt }\end{array}$ & $\begin{array}{c}\text { Anzahl in } \\
\text { der Studie }\end{array}$ & $\begin{array}{c}\text { Anzahl } \\
\text { ausgeschlossene Fälle }\end{array}$ & $\begin{array}{c}\text { \% weiblich } \\
\text { in der Studie }\end{array}$ & $\begin{array}{c}\text { \% männlich } \\
\text { in der Studie }\end{array}$ \\
\hline 1998 & 777 & 753 & $24(3.0 \%)$ & 52.3 & 47.7 \\
1999 & 741 & 716 & $25(3.4 \%)$ & 50.4 & 49.6 \\
2000 & 707 & 666 & $41(5.8 \%)$ & 52.3 & 47.7 \\
2001 & 810 & 769 & $41(5.1 \%)$ & 50.2 & 49.8 \\
2002 & 848 & 808 & $40(4.7 \%)$ & 50.2 & 49.8 \\
2003 & 846 & 787 & $59(7.0 \%)$ & 45.1 & 54.9 \\
2004 & 914 & 833 & $81(8.8 \%)$ & 51.4 & 48.6 \\
\hline Total & 5643 & 5332 & $311(5.5 \%)$ & 50.2 & 49.8 \\
\hline
\end{tabular}


50.2 Prozent der Stichprobe waren weiblichen Geschlechts. Der Anteil Mädchen schwankte in den verschiedenen Jahren zwischen $45-52 \%$, wobei die $\chi^{2}$-Differenztestung $\left(\chi^{2}=11.12\right.$, df $\left.=6, \mathrm{p}=.09\right)$ nicht statistisch signifikant ist. Die Prozentanteile der Geschlechter ist stabil, einzig 2003 sank der Anteil an Mädchen auf 45.1\%33.

\section{Instrumente}

Kriteriumsvariable

Übertrittsempfehlung der Lehrkräfte. Auf einer siebenstufigen Skala gaben die Lehrpersonen eine Zuweisungsempfehlung ab. Die Abstufungen waren folgendermassen: Progymnasium empfohlen (7), Progymnasium bedingt empfohlen (6), Sekundarschule empfohlen (5), Sekundarschule bedingt empfohlen (4), Realschule empfohlen (3), Realschule bedingt empfohlen (2), Werkklasse empfohlen (1). Wir bildeten aus den Angaben eine fünfstufige Skala, indem wir die Kategorien 1 bis 3 (bis zu Realschule empfohlen) zusammenfassten. Die Differenzierung zwischen einer für die Lehrperson «sicheren» Empfehlung und einer bedingten Empfehlung konnte in bisherigen Untersuchungen zu Übertrittsverfahren nicht gefunden werden.

\section{Prädiktorvariablen}

Standardisierter Leistungstest. Im Rahmen des Übertrittsverfahrens wird ein standardisierter Mathematik- und Deutschtest administriert. In beiden Testteilen können maximal 50 Punkte erzielt werden, so dass insgesamt maximal 100 Punkte erreicht werden können. Der Mathematiktest besteht aus den vier Subtests Grundoperationen, Textaufgaben, Brüche und Geometrie und der Deutschtest aus den Subtests Textschaffen, Textverständnis und Rechtschreibung. Die Aufgabeninhalte beziehen sich auf den Lehrplan der fünften und einen Teil der sechsten Klasse. Die Prüfungsaufgaben werden von Lehrpersonen der Sekundarstufe I und von nicht am Übertritt beteiligten Primarlehrpersonen auf der Basis der verbindlichen Lehrpläne erarbeitet. Zudem werden die Tests in einigen Klassen eines anderen Kantons, in welchem dieselben Lehrpläne gelten, validiert. Die Teilnahme am Leistungstest ist in der Regel für alle Schülerinnen und Schüler verpflichtend. Dispensiert werden heilpädagogisch begleitete Schülerinnen und Schüler mit grossen Entwicklungsrückständen. Der Leistungstest findet in allen Schulen am gleichen Tag statt, wird in den Klassenzimmern der Primarschule durchgeführt und von einer Lehrperson der Sekundarstufe I überwacht. Die Prüfungskorrekturen werden von Lehrpersonen der Sekundarstufe I durchgeführt, um die Reliabilität der Auswertungen zu garantieren. Die Testergebnisse werden für ganz Deutschfreiburg zentral ausgewertet.

\section{Verschiedene Formen der Beurteilung der Schulleistungen Lernprozessbeurteilung}

Am Ende des ersten Semesters der 6. Klasse wurden die Zeugnisbeurteilungen der Fächer Deutsch, Mathematik, Französisch und Naturkunde/Realien (natur- 
wissenschaftliche Fächer: Physik, Chemie und Naturlehre) berücksichtigt. Die Beurteilung ist dreistufig: (5) kommt bei der Erarbeitung der Lernziele gut voran, (3) hat bei der Erarbeitung der Lernziele Schwierigkeiten, (1) hat bei der Erarbeitung der Lernziele grosse Schwierigkeiten.

\section{Lernstandsbeurteilung}

Zudem wurde der Lernstand von den Lehrpersonen unmittelbar vor Abgabe der Lehrerempfehlung und der Übertrittsprüfung in den Fächern Deutsch, Mathematik, Französisch und Naturkunde/Realien beurteilt.

Die Beurteilung ist vierstufig: (7) hat Lernziele sehr gut erreicht, (5) hat Lernziele gut erreicht, (3) hat Lernziele erreicht, (1) hat Lernziele teilweise erreicht.

Damit ist dem heutigen Bestreben, standardorientierte Beurteilungen (vgl. Ditton \& Krüsken, 2006) anstelle der Noten, die sich an der Leistungsstärke der Klasse orientieren, als Erfolgskriterium zu berücksichtigen am ehesten Genüge getan. Zur Vergabe der globalen Ziffernnote pro Fach wurden die Lehrpersonen explizit angewiesen, diese nicht exakt nach dem arithmetischen Mittel der Prüfungsnoten $\mathrm{zu}$ vergeben, sondern die Leistungsstärke der Schülerin rsp. des Schülers holistisch einzuschätzen. Es wurden keine Ziffernnoten erfasst.

\section{Das Beobachtungs-Beurteilungsinstrument}

Die Lehrpersonen beurteilten anhand von 22 Items eines vorgegebenen Beobachtungsinstruments das Arbeits- und Lernverhalten und die allgemeinen kognitiven Fähigkeiten der Schülerinnen und Schüler auf einer dreistufigen Skala: beobachtetelbemerkte ich selten (1), beobachtetelbemerkte ich gelegentlich (2) und beobachtete/bemerkte ich oft (3). Entwickelt und validiert wurde dieses Beobachtungsinstrument von Schwegler (1989). Es wurde jedoch nachfolgend mehrmals ohne wissenschaftliche Begleitung verändert, so dass eine erneute Überprüfung der Struktur des Instruments notwendig wurde. Baeriswyl et al. (2006) hatten zu diesem Zweck mit den Daten des Jahrgangs 1999 eine Hauptkomponentenanalyse mit anschliessender Varimaxrotation durchgeführt. Die Inspektion des Scree-Plots (Eigenwerteverlauf der ersten vier Faktoren: 10.01, 1.87, 1.17, 0.94) sowie das Kriterium der inhaltlichen Interpretierbarkeit legten für die Daten des Jahrganges 1999 eine Zwei-Faktorenlösung nahe. Diese beiden Faktoren konnten in allen Jahrgängen (1998, sowie 2000-2004) repliziert werden. Auf dem ersten der beiden extrahierten Faktoren, von uns kognitive Fähigkeiten genannt, wiesen 10 Items (kann Handlungen gedanklich durchspielen; kann Gedankengänge klar darstellen; zeigt kritisches Denken; zeigt logisches, schlussfolgerndes Denken; erkennt Zusammenhänge; überträgt Gelerntes; fasst Neues rasch auf; erbringt gute Gedächtnisleistungen; findet eigene Lösungen; setzt angemessene Lerntechniken ein) eine hohe Ladung auf. Die aus den Items gebildete Skala hatte in den verschiedenen Jahren eine hohe interne Konsistenz (Cronbachs Alpha $=.93$ bis .94). Auf dem zweiten der beiden extrahierten Faktoren, von uns als schulische Motivation bezeichnet, hatten sechs Items (zeigt vielfältiges Inter- 
esse; lässt sich motivieren; führt Arbeiten sorgfältig aus; hat altersentsprechende Ausdauer; ist auch nach Misserfolg interessiert; arbeitet in Gruppen gut mit) ihre Primärladung. Die interne Konsistenz der gebildeten Skala war in den verschiedenen Jahren zufriedenstellend (Cronbachs Alpha $=.81$ bis .88 ).

\section{Statistische Analysen}

In Untersuchungen mit Schulklassen ist die Mehrebenenstruktur der gewonnenen Daten zu berücksichtigen, um mögliche Fehlschlüsse bei der Analyse zu vermeiden (Raudenbush \& Bryk, 2002). Durch das Klassenumfeld (dieselbe Lehrperson, denselben Unterricht, dieselbe Klasse) sind die Mitglieder eines Klassenverbandes einander in gewissen Belangen ähnlicher als den Kindern von anderen Klassen. Die statistische Annahme der Unabhängigkeit der Beobachtungseinheiten wird durch die Schachtelung von Schülerinnen und Schüler innerhalb von Klassen verletzt. Daher ist eine sorgfältige Korrektur der Schätzung der Standardfehler angebracht, um die Überschätzung von statistischer Signifikanz zu vermeiden. Da für Mehrebenenanalysen genügend Varianz auf den entsprechenden Ebenen auftreten muss (genügend Unterschiede zwischen den jeweiligen Clustern), gibt es Kennwerte, um abzuschätzen ob Mehrebenenanalysen angebracht sind, z.B. die Intra Class Correlation (ICC). Die ICC ist, im vorliegenden Fall, der Anteil der Gesamtvarianz, welcher zwischen den verschiedenen Klassen liegt. Ein weiteres Mass ist der Designeffekt. Er setzt die ICC in Beziehung zur durchschnittlichen Grösse der Cluster und sollte laut einer Muthén'schen Daumenregel über 2 liegen (Muthén \& Muthén, 1999) ${ }^{4}$. Nach Voranalysen und Abschätzung der ICC der abhängigen Variable Lehrerempfehlung, welche in allen Jahrgängen deutlich unter 5\% lag (ausser 2002: 6.8\%) und unter 2 liegenden Designeffekten (Muthén \& Muthén, 1999), wurde auf eine Modellierung von Prädiktoren auf der Klassenebene verzichtet. Zumal für die Analysen auf der Klassenebene Kontextvariablen nur spärlich vorhanden waren. Die Standardfehler jedoch wurden korrigiert, um dem durch die Schachtelung erzeugten Designeffekt Rechung zu tragen. Für diese Korrektur wurde im Programmpaket Mplus 4.1 die Option type = complex benutzt (Muthén \& Muthén 1998-2006). Für den Umgang mit fehlenden Werten wurde das in Mplus implementierte Full Information Maximum Likelihood Verfahren verwendet. Deskriptive Analysen wurden mit dem Programmpaket SPSS 16 durchgeführt.

\section{Ergebnisse und Diskussion}

\section{Deskriptive Befunde}

In Tabelle 2 werden die Mittelwerte und Korrelationen der Gesamtstichprobe 1998 bis 2004 (N=5332) berichtet. Ausser bei der Einschätzung der kognitiven Fähigkeiten ergaben sich statistisch signifikante Korrelationen zum Geschlecht (Mädchen=0). In der Regel waren diese Korrelationen negativ, d.h. die Mädchen 
wurden höher eingeschätzt. Eine Ausnahme bildete die Beurteilung des Lernstandes Mathematik dort resultierte eine geringe statistisch signifikante positive Korrelation. Erwartungsgemäss fanden sich hohe bis sehr hohe Korrelationen zwischen den Lernprozess-, den Lernstandsbeurteilungen, den kognitiven Fähigkeiten, der Vergleichsprüfung und der Lehrerempfehlung. Die Einschätzung der Motivation korreliert mit diesen Variablen etwas geringer, aber immer noch hoch.

Tabelle 2: Mittelwerte und Korrelationen der Gesamtstichprobe

\begin{tabular}{|c|c|c|c|c|c|c|c|c|c|c|c|c|c|c|c|c|}
\hline & $\mathrm{N}$ & $M$ & SD & Min & $\mathrm{Max}$ & 1 & 2 & 3 & 4 & 5 & 6 & 7 & 8 & 9 & 10 & $11 \quad 1$ \\
\hline $\begin{array}{ll} & \text { Geschlecht: } \\
(0=\mathrm{w}, 1=\mathrm{m})\end{array}$ & 5332 & 0.50 & .50 & 0 & 1 & & & & & & & & & & & \\
\hline 2 Math LP & 5272 & 4.01 & 1.30 & 1 & 5 & .03 & & & & & & & & & & \\
\hline 3 Deutsch LP & 5272 & 3.98 & 1.29 & 1 & 5 & -.18 & .56 & & & & & & & & & \\
\hline 4 Realien LP & 5299 & 4.37 & 1.10 & 1 & 5 & -.05 & .51 & .56 & & & & & & & & \\
\hline 5 Franz LP & 5289 & 4.10 & 1.31 & 1 & 5 & -.18 & .52 & .61 & .53 & & & & & & & \\
\hline 6 Math LS & 5248 & 4.75 & 1.89 & 1 & 7 & .04 & .81 & .56 & .49 & .51 & & & & & & \\
\hline 7 Deutsch LS & 5249 & 4.70 & 1.85 & 1 & 7 & -.18 & .55 & .81 & .53 & .58 & .65 & & & & & \\
\hline 8 Realien LS & 5288 & 5.31 & 1.75 & 1 & 7 & -.07 & .53 & .58 & .78 & .54 & .62 & .66 & & & & \\
\hline 9 Franz LS & 5272 & 4.98 & 1.94 & 1 & 7 & -.18 & .52 & .62 & .50 & .83 & .59 & .68 & .62 & & & \\
\hline $10 \begin{array}{l}\text { Kognitive } \\
\text { Fähigkeit }\end{array}$ & 5302 & 4.02 & 0.99 & 1 & 5 & -.02 & .71 & .68 & .60 & .61 & .74 & .71 & .67 & .66 & & \\
\hline 11 Motivation & 5300 & 4.38 & 0.76 & 1 & 5 & -.25 & .47 & .52 & .50 & .51 & .47 & .52 & .53 & .53 & .64 & \\
\hline $\begin{array}{l}\text { Vergleichs- } \\
\text { prüfung }\end{array}$ & 5273 & 63.9 & 16.7 & 6 & 98 & -.05 & .66 & .67 & .57 & .56 & .72 & .72 & .64 & .62 & .75 & .50 \\
\hline $\begin{array}{l}13 \begin{array}{l}\text { Lehrer- } \\
\text { empfehlung }\end{array}\end{array}$ & 5325 & 2.99 & 1.46 & 1 & 5 & -.09 & .68 & .71 & .57 & .63 & .78 & .80 & .70 & .72 & .80 & .56 \\
\hline
\end{tabular}

Anmerkung: 5\% Signifikanzniveau bei Koeffizienten $r>.03$

$\mathrm{LP}=$ Beurteilung Lernprozess, LS = Beurteilung Lernstand

In der Tabelle 3 werden die Mittelwerte und Standardabweichungen pro Erhebungsjahr berichtet. Einzig die Mittelwerte der Vergleichsprüfung unterscheiden sich hoch signifikant. Ebenfalls signifikante Unterschiede finden sich in drei Leistungsbeurteilungen, in der Mathematik sowohl die Lernprozess- wie die Lernstandsbeurteilung und in Französisch die Lernstandsbeurteilung. Ein signifikanter Haupteffekt ist bei der Beurteilung der kognitiven Fähigkeit feststellbar. Die multiplen Mittelwertsvergleiche nach Scheffé zeigen jedoch, dass keiner dieser signifikanten Haupteffekte in den einzelnen Vergleichen signifikant ist.

Die Verteilung der Zuweisung zu den verschiedenen Abteilungen (Tabelle 4) unterscheidet sich im Verlauf der sieben Jahre nicht statistisch signifikant $\left(\chi^{2}=\right.$ $34.60, d f=24, \mathrm{p}=.08)$. In der standardmässigen Auswertung des Übertrittverfahrens ist ersichtlich, dass sich der beobachtbare Trend zu einem höheren Anteil an Progymnasialempfehlungen in den Jahren 2005-2008 wieder gelegt hat. 
Tabelle 3: Mittelwerte der Prädiktoren während der Erhebungsjahre

\begin{tabular}{|c|c|c|c|c|c|c|c|c|c|c|c|c|c|c|c|}
\hline & \multicolumn{2}{|c|}{1998} & \multicolumn{2}{|c|}{1999} & \multicolumn{2}{|c|}{2000} & \multicolumn{2}{|c|}{2001} & \multicolumn{2}{|c|}{2002} & \multicolumn{2}{|c|}{2003} & \multicolumn{2}{|c|}{2004} & \multirow{2}{*}{$\begin{array}{l}\text { ANOVA } \\
\text { Sign. }\end{array}$} \\
\hline & M & $\mathrm{SD}$ & M & $\mathrm{SD}$ & M & SD & M & SD & M & SD & M & SD & M & SD & \\
\hline Math LP & 3.93 & 1.34 & 3.95 & 1.32 & 3.97 & 1.31 & 4.02 & 1.30 & 4.06 & 1.26 & 4.00 & 1.30 & 4.14 & 1.29 & * \\
\hline Deutsch LP & 3.93 & 1.29 & 3.98 & 1.31 & 3.88 & 1.33 & 4.00 & 1.28 & 4.03 & 1.29 & 3.96 & 1.23 & 4.05 & 1.26 & \\
\hline Realien LP & 4.40 & 1.05 & 4.35 & 1.09 & 4.29 & 1.15 & 4.38 & 1.11 & 4.40 & 1.09 & 4.38 & 1.07 & 4.37 & 1.12 & \\
\hline Franz LP & 4.05 & 1.34 & 4.13 & 1.26 & 4.08 & 1.33 & 4.11 & 1.33 & 4.08 & 1.33 & 4.05 & 1.30 & 4.21 & 1.28 & \\
\hline Math LS & 4.69 & 1.93 & 4.67 & 1.86 & 4.65 & 1.86 & 4.79 & 1.87 & 4.67 & 1.91 & 4.77 & 1.91 & 4.96 & 1.89 & $*$ \\
\hline Deutsch LS & 4.65 & 1.87 & 4.72 & 1.82 & 4.63 & 1.88 & 4.75 & 1.87 & 4.66 & 1.85 & 4.68 & 1.85 & 4.83 & 1.82 & \\
\hline Realien LS & 5.43 & 1.71 & 5.30 & 1.71 & 5.21 & 1.79 & 5.37 & 1.72 & 5.18 & 1.83 & 5.34 & 1.76 & 5.35 & 1.71 & \\
\hline Franz LS & 4.89 & 1.97 & 4.99 & 1.86 & 4.95 & 1.97 & 5.06 & 1.91 & 4.84 & 1.96 & 4.97 & 1.95 & 5.15 & 1.92 & * \\
\hline $\begin{array}{l}\text { Kognitive } \\
\text { Fähigkeit }\end{array}$ & 3.99 & 0.94 & 4.00 & 0.97 & 3.98 & 1.02 & 4.07 & 0.99 & 3.96 & 1.04 & 4.04 & 1.00 & 4.10 & 1.00 & $*$ \\
\hline Motivation & 4.37 & 0.72 & 4.36 & 0.75 & 4.35 & 0.77 & 4.38 & 0.79 & 4.39 & 0.79 & 4.36 & 0.75 & 4.45 & 0.72 & \\
\hline $\begin{array}{l}\text { Lehrer- } \\
\text { empfehlung }\end{array}$ & 2.89 & 1.42 & 2.94 & 1.41 & 2.97 & 1.44 & 3.04 & 1.44 & 3.00 & 1.48 & 3.01 & 1.49 & 3.06 & 1.49 & \\
\hline $\begin{array}{l}\text { Vergleichs- } \\
\text { prüfung }\end{array}$ & 60.3 & 16.7 & 63.4 & 15.9 & 64.6 & 16.7 & 66.4 & 16.6 & 62.5 & 16.5 & 67.0 & 16.9 & 63.3 & 16.6 & $* * *$ \\
\hline
\end{tabular}

Anmerkung: LP = Beurteilung Lernprozess, LS = Beurteilung Lernstand ${ }^{* * *}=\mathrm{p}<.001,{ }^{* *}=\mathrm{p}<.01,{ }^{*}=\mathrm{p}<.05$

Tabelle 4: Verteilung der Übertrittsempfehlungen auf die Schulabteilungen (Prozentangaben)

\begin{tabular}{|ccccccc|}
\hline Jahrgang & $\mathrm{N}$ & $\begin{array}{c}\text { Werkklasse/ } \\
\text { Realschule }\end{array}$ & $\begin{array}{c}\text { Sekundar- } \\
\text { schule bedingt }\end{array}$ & $\begin{array}{c}\text { Sekundar- } \\
\text { schule }\end{array}$ & $\begin{array}{c}\text { Progymna- } \\
\text { sium bedingt }\end{array}$ & $\begin{array}{c}\text { Progymna- } \\
\text { sium }\end{array}$ \\
\hline 1998 & 752 & 25.5 & 10.2 & 35.0 & 8.6 & 20.6 \\
1999 & 715 & 22.9 & 11.2 & 35.9 & 8.4 & 21.5 \\
2000 & 666 & 22.8 & 12.6 & 33.6 & 6.9 & 24.0 \\
2001 & 767 & 22.4 & 9.3 & 34.7 & 8.9 & 24.8 \\
2002 & 806 & 24.4 & 10.4 & 31.1 & 8.9 & 25.1 \\
2003 & 786 & 24.2 & 11.7 & 29.5 & 7.9 & 26.7 \\
2004 & 833 & 24.6 & 8.0 & 31.3 & 9.1 & 26.9 \\
$1998-04$ & 5325 & 23.9 & 10.4 & 32.9 & 8.4 & 24.3 \\
\hline
\end{tabular}

Die durchschnittliche Übereinstimmung zwischen Lehrer- und Elternempfehlung beträgt 1998 bis $200480.7 \%$ (78.2 bis 83.2\%) ( $\chi^{2}=10.97$, $\mathrm{df}=6, \mathrm{p}=$ .09). Dabei beläuft sich der grösste Teil der Abweichungen bei den Empfehlungen auf eine halbe Stufe (z.B. Elternempfehlung: Sekundarabteilung empfohlen und Lehrerempfehlung: Sekundarabteilung bedingt empfohlen). Im Durchschnitt geben $4.3 \%$ der Eltern in dieser Zeitspanne eine um eine Abteilung höhere Einstufung an (z.B. Elternempfehlung: Sekundarabteilung; Lehrerempfeh- 
lung: Realabteilung). Dieser Prozentsatz der Nicht-Übereinstimmung zwischen Eltern- und Lehrerempfehlung streut zwischen 3.4\% und 5.8\%. Zwischen 1998 und 2004 hat diese Abweichung zugenommen, wobei sie in den Jahren 20052007 wieder abgenommen hat. Nur $0.4 \%$ der Eltern geben eine um eine Abteilung tiefere Einstufung. Diese hohe Übereinstimmung ist neben dem ausgeprägten Kommunikationsprozess, auch hoch, weil alle Beteiligten um die Durchführung des objektiven Leistungsvergleichs wissen. Auf Grund der hohen Übereinstimmung wurde bei den folgenden regressionsanalytischen Modellen die Elternempfehlung nicht berücksichtigt.

\section{Ergebnisse zur ersten Fragestellung: Ist die Bildung der übertrittsempfehlung über die sieben Jahre hinweg konsi- stent?}

Um zu untersuchen, ob die Übertrittsempfehlung über die sieben Jahre ähnlich gebildet wird, wurde für jedes Jahr das gleiche Prognose-Modell gerechnet.

Die berücksichtigten Variablen für die Erklärung der Zuweisungsempfehlung der Lehrpersonen sind: das Geschlecht, die Lernprozessbeurteilung (am Ende des 1. Semesters der 6. Klasse) in den Fächern Mathematik, Deutsch, Französisch und Naturkunde/Realien, die Lernstandsbeurteilung in diesen Fächern (kurz vor der Vergleichsprüfung und der Abgabe der Übertrittsempfehlung), die Beurteilung der kognitiven Fähigkeiten und der Motivation, sowie die Testleistung an der Vergleichsprüfung. Als Datengrundlage für die Modelle wurden die Skalenwerte unstandardisiert und unzentriert verwendet, nur die Vergleichsprüfung wurde pro Jahr z-standardisiert (Da der Mittelwert der Prüfung über die Jahre hinweg variiert diente dies dazu, die Vergleichbarkeit über die Jahre hinweg zu erhöhen).

Der Modellfit ist für jedes der Jahre 1999 bis 2004 befriedigend und einzig für 1998 etwas kritisch. Das Geschlecht, die Lernprozessbeurteilungen und die Einschätzung der Motivation sind weniger starke Prädiktoren, als die Lernstandsbeurteilung, die Einschätzung der kognitiven Fähigkeiten und die Vergleichsprüfung. Ein leichter Geschlechtseffekt findet sich einzig im Jahr 1998, wobei die Mädchen unter Kontrolle der erwähnten Variablen von den Lehrpersonen leicht höhere Empfehlungen erhalten. Dies ist auch das einzige Jahr in dem die Motivation ein signifikant negativer Prädiktor ist. Die aufgeklärte Varianz der Übertrittsempfehlung variiert zwischen $81 \%$ und 83\%.

Um zu untersuchen, ob sich die Genese der Lehrerempfehlung während der verschieden Jahre unterscheidet, wurde überprüft, ob sich die Vorhersage der Lehrerempfehlung in den verschiedenen Jahren signifikant unterscheidet (vgl. Tabelle 5). 
Tabelle 5: Vergleich der Vorhersage der Lehrerempfehlung in den Jahren 1998-2004

\begin{tabular}{|c|c|c|c|c|c|c|c|c|c|c|c|c|c|c|}
\hline & \multicolumn{2}{|c|}{1998} & \multicolumn{2}{|l|}{1999} & \multicolumn{2}{|l|}{2000} & \multicolumn{2}{|l|}{2001} & \multicolumn{2}{|l|}{2002} & \multicolumn{2}{|l|}{2003} & \multicolumn{2}{|c|}{2004} \\
\hline $\begin{array}{l}\text { Geschlecht: } \\
(0=\mathrm{w}, 1=\mathrm{m})\end{array}$ & -.04 & * & -.01 & & .00 & & -.03 & & -.01 & & .03 & & .00 & \\
\hline Math LP & .01 & & -.04 & & .03 & & -.02 & & .01 & & -.02 & & .02 & \\
\hline Deutsch LP & .04 & & -.01 & & .05 & & -.01 & & .02 & & .09 & * & -.03 & \\
\hline Realien LP & -.02 & & .02 & & -.06 & & -.01 & & -.03 & & -.07 & * & -.01 & \\
\hline Franz LP & .01 & & -.02 & & -.03 & & .03 & & -.02 & & .00 & & -.07 & $*$ \\
\hline Math LS & .26 & $* * *$ & .28 & *** & .21 & *** & .22 & *** & .20 & *** & .25 & *** & .25 & *** \\
\hline Deutsch LS & .26 & $* * *$ & .25 & *** & .25 & *** & .28 & *** & .18 & *** & .25 & *** & .28 & *** \\
\hline Realien LS & .08 & $* *$ & .05 & & .11 & $* *$ & .10 & ** & .09 & ** & .11 & ** & .08 & * \\
\hline Franz LS & .13 & $* *$ & .11 & ** & .14 & *** & .09 & $* *$ & .18 & *** & .12 & * & .24 & *** \\
\hline $\begin{array}{l}\text { Kognitive } \\
\text { Fähigkeit }{ }^{1}\end{array}$ & .21 & *** & .25 & *** & .17 & *** & .27 & $* * *$ & .25 & *** & .24 & $* *$ & .15 & ** \\
\hline Motivation ${ }^{1}$ & -.09 & ** & .03 & & .01 & & -.06 & & .01 & & .01 & & -.03 & \\
\hline $\begin{array}{l}\text { Vergleichs- } \\
\text { prüfung }{ }^{2}\end{array}$ & .17 & *** & .12 & *** & .18 & *** & .14 & $* * *$ & .16 & *** & .05 & & .16 & *** \\
\hline$R^{2}$ & .83 & & .82 & & .83 & & .82 & & .83 & & .81 & & .83 & \\
\hline CFI & .89 & & .92 & & .93 & & .95 & & .95 & & .95 & & .95 & \\
\hline TLI & .87 & & .91 & & .92 & & .94 & & .94 & & .94 & & .94 & \\
\hline RMSEA & .06 & & .05 & & .05 & & .04 & & .04 & & .04 & & .04 & \\
\hline SRMR & .05 & & .04 & & .04 & & .04 & & .03 & & .04 & & .03 & \\
\hline
\end{tabular}

Anmerkungen: Berichtet sind standardisierte Werte

${ }^{* * *}=\mathrm{p}<.001,{ }^{* *}=\mathrm{p}<.01,{ }^{*}=\mathrm{p}<.05, R^{2}=$ aufgeklärte Varianz

${ }^{1}$ Latent modelliert, ${ }^{2}$ pro Jahr z-standardisiert; LP = Beurteilung Lernprozess, LS = Beurteilung Lernstand

Im ersten Schritt wurde die metrische Messinvarianz der latenten Variablen in den verschiedenen Jahren überprüft, und konnte bestätigt werden ${ }^{4}$ (Byrne, 2000). Im zweiten Schritt wurde dieses Modell mit einem Modell verglichen in dem die Kovarianzen der Prädiktoren über die verschiedenen Jahre gleichgesetzt wurden. So kann man testen, ob sich diese Kovarianzen über die Jahre hinweg statistisch signifikant unterscheiden. Dies ist eine Bedingung, damit man anschliessend die Prädiktoren der Regression gleichsetzten kann. Mittels skaliertem $\chi^{2}$-Differenztest wurde verglichen, ob sich der Fit dieses restriktiveren Modells mit gleichgesetzten Kovarianzen signifikant verschlechtert (auf Grund der geschachtelten Daten wurde das Verfahren MLR ${ }^{6}$ verwendet, vgl. Muthen \& Muthen, 2005). Diese Modelle unterscheiden sich nicht signifikant voneinander (skalierte $\chi^{2}$-Differenz $=255.9, d f=378$, n.s.). Anschliessend wurde dieses Modell mit dem Modell verglichen in dem die Regressionskoeffizienten der Prädiktoren über die Jahre hinweg jeweils gleichgesetzt wurden (z.B. gleicher Regres- 
sionskoeffizient von Geschlecht im Jahr 1998, 1999 ... 2004; gleicher Regressionskoeffizient von kognitiven Fähigkeiten im Jahr 1998, 1999 ... 2004 usw.). Dadurch wurde getestet, ob sich die Regressionsgewichte über die Jahre hinweg unterscheiden, d.h. ob diese Prädiktoren signifikant unterschiedlich stark mit der Lehrerempfehlung zusammenhängen. Die Anpassungsgüte dieses Modell verschlechterte sich durch die einschränkende Gleichsetzung der Regressionskoeffizienten nicht signifikant (skalierte $\chi^{2}$-Differen $z=43, d f=72$, n.s.). Die verschiedenen Jahre unterscheiden sich statistisch nicht signifikant $d$. h. die Determinanten für die Bildung der Lehrerempfehlung wurden über die Jahre hinweg nicht statistisch signifikant unterschiedlich gewichtet.

\section{Diskussion zur ersten Fragestellung}

Weil der grösste Teil der Lehrerschaft eine Klasse während der fünften und sechsten Klasse unterrichtet, also nur jedes zweite Jahr eine Klasse ins Übertrittsverfahren führt, und weil keine Richtlinien oder Empfehlungen zur Bildung der Übertrittsempfehlung bestehen, konnte diese zeitlich stabile Modellierung der Lehrerempfehlung nicht unbedingt erwartet werden. Die Lehrpersonen stützen sich bei der Urteilsbildung vor allem auf die Lernstandsbeurteilungen, d.h. die Einschätzung der Fachleistungen, bezogen auf das Erreichen der Lernziele und weniger auf die Lernfortschrittsbeurteilung. Für die Schulleistungsbeurteilung beziehen sie sich demnach stärker auf eine Kriteriums- oder Sachnorm (Klauer, 1987) als auf die Individualnorm (Rheinberg, 2006). Zudem basieren sie sich nicht nur auf die geprüften Fächer Mathematik und Deutsch, sondern auch auf die Französisch- und Naturkundeleistungen. Die Einschätzung der kognitiven Fähigkeiten ist ebenfalls ein konstant wichtiger Faktor, der zusätzlich zur Leistungsbeurteilung das Konstrukt der Lehrerempfehlung aufklärt. Etwas überraschend erscheint hingegen, dass die Einschätzung der Motivation das Modell nur 1998 signifikant mitbestimmt, und die Motivation sich sogar noch negativ auf die Lehrerempfehlung auswirkt. Dies könnte jedoch mit dem Geschlechtseffekt zusammenhängen. Mit der Ausnahme von 2003 ist das Resultat der Vergleichsprüfung ein zusätzlicher Prädiktionsfaktor.

\section{Ergebnisse zur zweiten Fragestellung: Welche Beurtei- lungsform trägt wie viel zur Bildung der Lehrerempfeh- lung bei?}

Von besonderem Interesse war der Vergleich der Lernprozess- mit der Lernstandseinschätzung. Die Lernprozessbeurteilung weist eher auf einen Individualnormbezug und die Lernstandsbeurteilung auf die Sach- oder Kriteriumsnorm hin. Nimmt eine der beiden Beurteilungen mehr Einfluss auf die Lehrerempfehlung? Wird die Lehrerempfehlung auf Grund des aktuellen Leistungsstandes gemacht, oder werden auch Lernprozessentwicklungen berücksichtigt? Haben Mädchen und Jungen die gleichen Chancen? Welche Rollen spielen die Lehrerbeurteilungen der kognitiven Fähigkeiten und der Motivation in der Genese der 
Lehrerempfehlung? Weil die Prädiktionsmodelle der Lehrerempfehlung über die Jahre hinweg als konsistent betrachtet werden können, wurden die nachfolgenden Analysen am Gesamtdatensatz von 1998 bis 2004 vorgenommen.

Die in Tabelle 5 dargestellten Resultate zeigten bereits, dass die Lernprozessbeurteilung weniger prädiktiv ist, als die Lernstandsbeurteilung. In den Modellen der Tabelle 6 werden nun die Daten der Gesamtstichprobe genutzt und die Indikatorenkomplexe der Bildung des Lehrerurteils werden schrittweise zur Prädiktion der Lehrerempfehlung eingeführt.

Tabelle 6: Aufschlüsselung der Lehrerempfehlung mit den Gesamtdaten der Jahre 1998-2004

\begin{tabular}{|c|c|c|c|c|c|c|c|c|c|c|}
\hline & \multicolumn{2}{|c|}{ Modell 1} & \multicolumn{2}{|c|}{ Modell 2} & \multicolumn{2}{|c|}{ Modell 3} & \multicolumn{2}{|c|}{ Modell 4} & \multicolumn{2}{|c|}{ Modell 5} \\
\hline Geschlecht: $(0=w, 1=m)$ & .00 & & .01 & & .01 & & -.01 & & -.01 & \\
\hline Math LP & .34 & $* * *$ & & & .06 & *** & .01 & & .00 & \\
\hline Deutsch LP & .36 & $* * *$ & & & .07 & *** & .03 & ** & .02 & \\
\hline Realien LP & .11 & $* * *$ & & & .00 & & -.02 & & -.03 & * \\
\hline Franz LP & .17 & $* * *$ & & & .00 & & -.01 & & -.01 & \\
\hline Math LS & & & .37 & $* * *$ & .32 & $* * *$ & .26 & $* * *$ & .23 & $* * *$ \\
\hline Deutsch LS & & & .37 & $* * *$ & .32 & $* * *$ & .28 & $* * *$ & .25 & $* * *$ \\
\hline Realien LS & & & .12 & $* * *$ & .11 & $* * *$ & .09 & $* * *$ & .08 & $* * *$ \\
\hline Franz LS & & & .18 & $* * *$ & .17 & $* * *$ & .15 & $* * *$ & .14 & $* * *$ \\
\hline Kognitive Fähigkeit ${ }^{1}$ & & & & & & & .25 & $* * *$ & .21 & $* * *$ \\
\hline Motivation ${ }^{1}$ & & & & & & & -.02 & & -.01 & \\
\hline Vergleichsprüfung ${ }^{2}$ & & & & & & & & & .15 & $* * *$ \\
\hline$R^{2}$ & .66 & & .80 & & .80 & & .81 & & .82 & \\
\hline CFI & 1 & & 1 & & 1 & & .95 & & .95 & \\
\hline TLI & 1 & & 1 & & 1 & & .94 & & .94 & \\
\hline RMSEA & 0 & & 0 & & 0 & & .04 & & .04 & \\
\hline SRMR & 0 & & 0 & & 0 & & .03 & & .03 & \\
\hline
\end{tabular}

Anmerkungen: Berichtet sind standardisierte Werte

${ }^{* * *}=\mathrm{p}<.001,{ }^{* *}=\mathrm{p}<.01,{ }^{*}=\mathrm{p}<.05, \mathrm{R}^{2}=$ aufgeklärte Varianz

${ }^{1}$ Latent modelliert, ${ }^{2}$ pro Jahr z-standardisiert; LP $=$ Beurteilung Lernprozess, LS = Beurteilung Lernstand

Im ersten Modell (M1) fliessen die Beurteilungen des Lernprozesses (kommt bei der Erarbeitung der Lernziele gut voran ...) ein und erklären 66\% der Varianz. Mathematik und Deutsch sind die stärksten Prädiktoren, wobei Naturkunde und Französisch auch statistisch signifikant sind. Im zweiten Modell (M2) werden nur die Lernstandsbeurteilungen (erreicht die Lernziele gut ...) berücksichtigt, wobei ebenfalls alle Fächer statistisch signifikant sind, wiederum mit Mathematik und Deutsch als stärkste Prädiktoren. Die aufgeklärte Varianz wird mit der Lernstandsbeurteilung (im Vergleich zur Lernprozessbeurteilung) beachtlich von 66\% auf 80\% erhöht. Das dritte Modell (M3) kombiniert die Lernprozessund die Lernstandsbeurteilungen. Die Lernstandsbeurteilungen bleiben allesamt 
statistisch signifikant, wobei von den Lernprozessbeurteilungen nur Mathematik und Deutsch statistisch signifikant sind, jedoch nicht zu einem Anstieg der erklärten Varianz beitragen, sondern zu einem Absinken der Koeffizienten der Lernstandsbeurteilung führen. Im vierten Modell (M4) werden die Lehrpersoneneinschätzung der kognitiven Fähigkeiten und der Motivation zugefügt. Die Einschätzung der kognitiven Fähigkeiten ist ein fast so starker Prädiktor wie die Beurteilung von Mathematik und Deutsch, die anderen Prädiktoren sinken dementsprechend ab. Die Motivation zeigt keine signifikante Wirkung. Im fünften Modell (5) wird die Prüfung hinzugefügt und wird ebenfalls ein statistisch signifikanter Prädiktor. Die Prüfung findet real-zeitlich nach Abgabe der Übertrittsempfehlung statt. Auf das reale Entscheidungsmodell der Lehrpersonenempfehlung hat sie keinen Einfluss. Dennoch stellt sich die Frage, ob das Prüfungsergebnis, zusätzlich zu den Variablen, die der Lehrperson als Informationsquelle zur Verfügung stehen, einen weiteren Anteil der Übertrittsempfehlung zu erklären vermag. Sie weist, unter Kontrolle aller anderen Variablen, einen statistisch signifikanten zusätzlichen prädiktiven Wert auf. Die erklärte Varianz erhöht sich um $1 \%$ auf $82 \%$.

\section{Diskussion der zweiten Fragestellung}

Die Lehrerempfehlung für den Übertritt in die Sekundarstufe I wird durch das Geschlecht nicht beeinflusst. Die eher kriteriumsnorm-bezogene Lernstandsbeurteilung klärt die Varianz der Lehrerempfehlung beachtlich mehr auf als die Lernprozessbeurteilung. Diese starke Fokussierung der Lehrpersonen auf die Anforderungen des Lehrplans ist möglicherweise darauf zurückzuführen, dass die Aufgaben der Vergleichsprüfung strikt nach dem Lehrplan aufgebaut sind. Der Unterschied vom Modell 3 zu Modell 4 lässt vermuten, dass die Einschätzung der kognitiven Fähigkeiten stark auf Beobachtungen zum Lernfortschritt oder zum Lernprozess in der Mathematik beruht. Eher Widererwarten bleibt die Einschätzung der Motivation ohne zusätzlichen Einfluss auf die Lehrerempfehlung. Beachtenswert ist die Lernprozessbeurteilung in Deutsch als zusätzliche Informationsquelle zu den Lernstandsbeurteilungen im Modell 4, die im Modell 5 unter Hinzunahme der Vergleichsprüfung unter die Signifikanzgrenze absinkt. Modell 4 kann als das realistische Prädiktionsmodell der Lehrerempfehlung bezeichnet werden, weil hier sämtliche Faktoren berücksichtigt werden, welche die Lehrperson zur Bildung ihrer Übertrittsempfehlung hat. Die Lernstandsbeurteilungen in Deutsch und Mathematik, sowie die Einschätzung der kognitiven Fähigkeiten sind dabei die stärksten Prädiktoren. Die Varianzaufklärung kann mit dem Beobachtungs- Beurteilungsinstrument lediglich um 1\% auf $81 \%$ erhöht werden. Der grösste Teil der Varianzaufklärung liegt in der Lernstandsbeurteilung. Mit dem Modell 5 wird das reale Empfehlungsmodell überschritten, weil die Vergleichsprüfung zeitlich nach der Abgabe der Lehrerempfehlung stattfindet. Es zeigt sich dennoch, dass die Vergleichsprüfung zur schulischen Beurteilung zusätzliche Information enthält. 


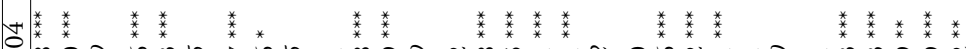

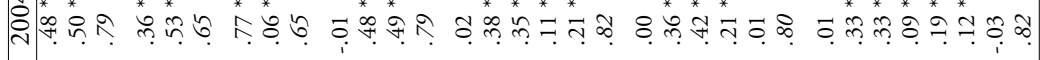

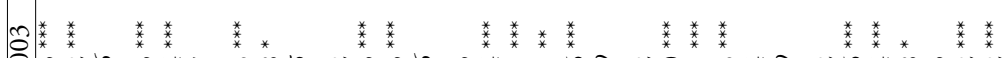

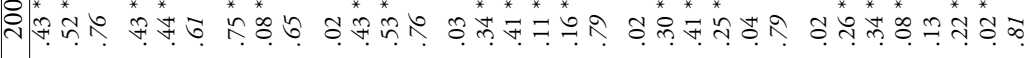

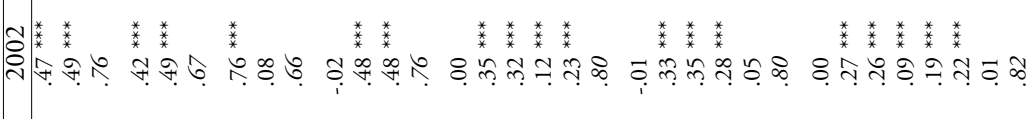

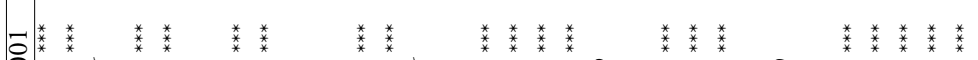

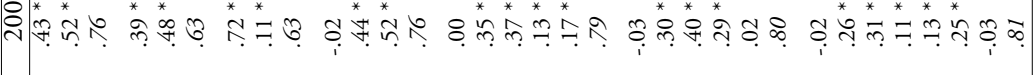

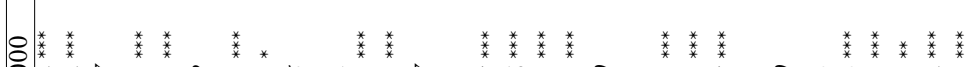

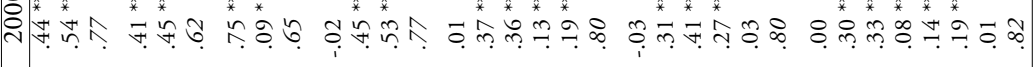

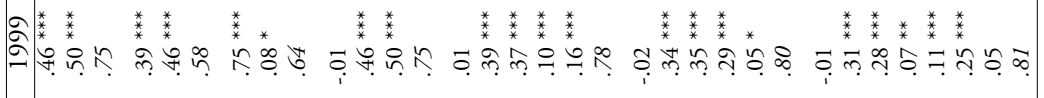

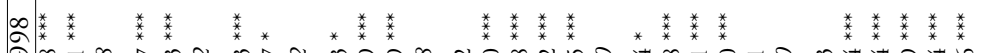

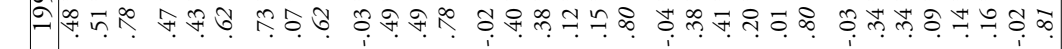

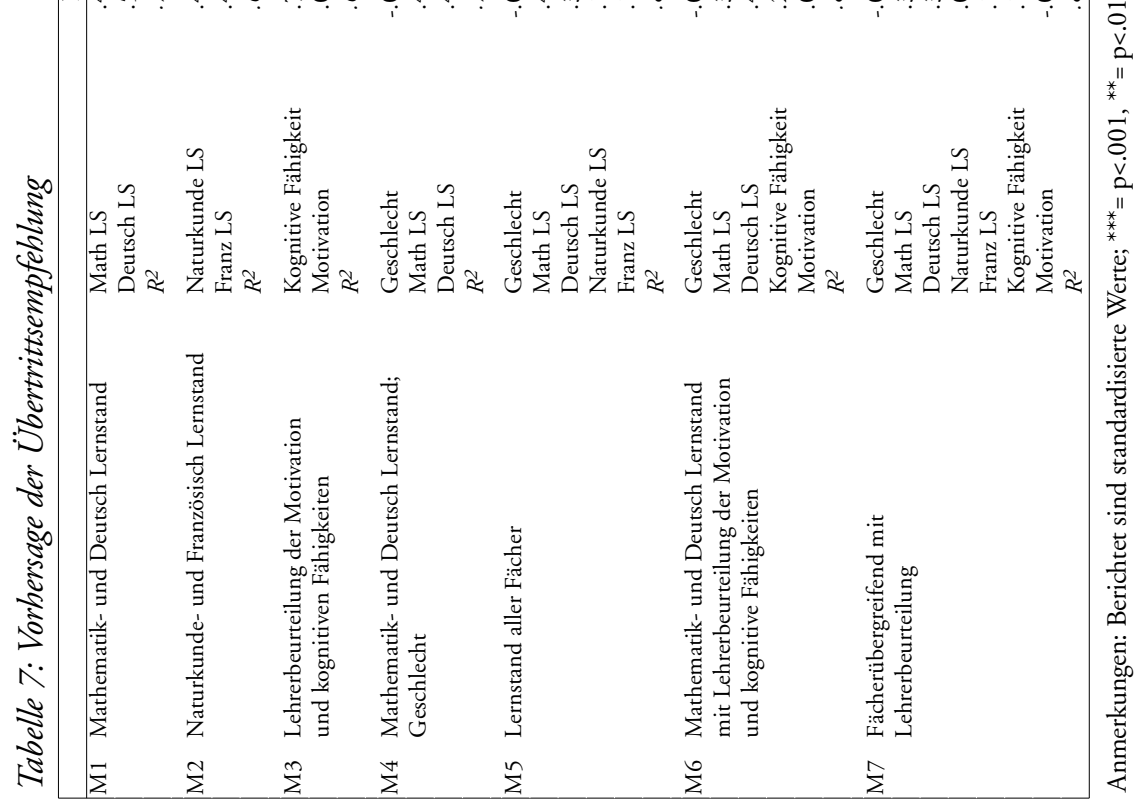


Ergebnisse zur dritten Fragestellung: Inwiefern wird die übertrittsempfehlung durch die fachspezifische Lernstandsbeurteilung beeinflusst?

In bisherigen Forschungen wird berichtet, die Deutschnote hätte für die Lehrerempfehlung eine grössere Bedeutung als die Mathematiknote (Bos et al., 2004; Stubbe \& Bos, 2008).

Um genauer zu determinieren, wie stark die Fächer gewichtet werden (vgl. Tabelle 7) wird die Lernprozessbeurteilung in den folgenden Modellen nicht berücksichtigt, weil sich gezeigt hat, dass sie für die Übertrittsempfehlung eine weniger wichtige Rolle als die Lernstandsbeurteilung spielt. In Modell 1 (M1) wird die Mathematik- und Deutschlernstandsbeurteilung berücksichtigt. In allen Jahren ist die Deutschbeurteilung ein stärkerer Prädiktor als die Mathematikbeurteilung. Durch die beiden Prädiktoren werden zwischen 75\% und 79\% der Varianz aufgeklärt. In Modell 2 (M2) werden die Naturkunde- und Französischnoten als Prädiktoren berücksichtigt. Im zweisprachigen (de/fr) Kanton Freiburg, wird erwartungsgemäss dem Fach Französisch in sechs von sieben Jahren mehr Gewicht beigemessen als der Naturkunde. In Modell 3 (M3) zeigt sich, dass die Lehrerbeurteilung der kognitiven Fähigkeiten viel stärker ins Gewicht fällt, als die Beurteilung der Motivation. Die Hinzunahme des Geschlechts in Modell 4 (M4) zeigt keine grösseren Einflüsse. In Modell 5 (M5) werden alle vier Fächer als signifikante Prädiktoren berücksichtigt. Kontrolliert für Französisch- und Naturkundenoten sind Mathematik und Deutsch mit etwa gleich starker Gewichtung die bedeutendsten Prädiktoren. Diese Angleichung von Mathematik und Deutsch findet in Modell 6 (M6) unter Berücksichtigung von Motivation und kognitiven Fähigkeiten nicht statt. Unter Beizug der kognitiven Fähigkeiten sinkt die Prädiktionskraft der Lernstandsbeurteilung von Mathematik. Modell 7 (M7) zeigt die Komplexität des Zusammenspiels der verschiedenen Faktoren. Diese tendenziellen Unterschiede zwischen den Jahren sind statistisch nicht signifikant unterschiedlich (skalierte $\chi^{2}$-Differenz $=32, d f=42$, n.s. $)^{7}$. Zusätzlich wurde überprüft, ob die Fächer Deutsch und Mathematik unterschiedlich stark in die Lehrerempfehlung einfliessen. Das Modell 7 wurde verglichen mit einem Modell in dem die Regressionsgewichte dieser beiden Fächer gleichgesetzt wurden. Der Fit des Modells verschlechterte sich nicht signifikant $\left(\chi^{2}-\right.$ Differenz $=3.13, d f=1$, n.s.). Die Fächer werden nicht statistisch signifikant unterschiedlich gewichtet.

\section{Diskussion der dritten Fragestellung}

In Bezug auf die Gewichtung der Prädiktoren zeigte sich tendenziell, dass Deutsch meistens stärker als Mathematik oder ebenso stark gewichtet wurde. Dieser augenscheinliche Unterschied konnte jedoch statistisch nicht bestätigt werden. Resultate anderer Studien (Bos et al., 2004; Stubbe \& Bos, 2008), welche Deutsch als stärkeren Prädiktor als Mathematik berichten, konnten somit für dieses Übertrittsverfahren in einer längsschnittlichen Überprüfung nicht be- 
stätigt werden. In gewissen Jahren wurden die beiden Fächer fast gleich stark gewichtet, in gewissen Jahren traten tendenzielle Unterschiede auf. Lernstandsbeurteilung von Fächern wie Naturkunde und Französisch, sowie den Lehrerbeurteilungen von kognitiven Fähigkeiten bergen zusätzliche Informationen. Diese zusätzlichen Informationen verändern die Gewichtung der Mathematik und Deutschnote. Die Einschätzung der Motivation trägt, unter der Berücksichtigung der anderen Prädiktoren, fast nie zusätzliche Information bei. Die Varianzaufklärung dieser beiden Fächer von 75 - 89\% ist vergleichbar mit den Untersuchungen von Stahl (2007) und Thiel (2006), und höher als die von Stubbe und Bos (2008) berichteten 55\%.

\section{Gesamtdiskussion}

Die Verfügbarkeit von Daten aus sieben aufeinander folgenden Übertrittsjahren hat eine zuverlässige Erforschung der Lehrerempfehlung ermöglicht und so konnte die Frage nach der Konsistenz der Determinanten der Lehrerempfehlung bearbeitet werden. Diese Frage betrifft einen recht engen Ausschnitt der Übertrittsthematik, dieser ist aber unserer Meinung nach ein wichtiger Aspekt der Gerechtigkeit von Übertrittsverfahren. Es zeigte sich, dass es zwar immer wieder tendenziell leichte Unterschiede in der Gewichtung von Determinanten gibt, dass sich die Modelle insgesamt allerdings über die Jahre hinweg, trotz der veränderten Lehrerschaft, nicht statistisch signifikant voneinander unterscheiden. Die Beurteilungsgerechtigkeit über die Jahre hinweg ist diesbezüglich gewährleistet.

Auch bei dieser scheinbar guten Repräsentativität der Lehrerempfehlung, muss darauf aufmerksam gemacht werden, dass Resultate, auf Grund der Daten eines einzigen Jahrganges vorsichtig aufgenommen werden müssen (vgl. z.B. Geschlechtseffekt oder Einfluss von Motivation in Tabelle 5).

Erfreulich ist, dass in der Regel bei den Lehrpersonen keine direkte Bevorteilung eines Geschlechtes auftritt. Die Daten mehrerer Jahre relativieren zum Teil Untersuchungsergebnisse, welche auf der Analyse eines einzigen Jahrgangs beruhen.

Im Vergleich von Lernprozess- und Lernstandsbeurteilungen zeigte sich, dass die Lehrerempfehlung stärker auf der Lernstandsbeurteilung basiert. Dies kann als zuverlässiges Resultat interpretiert werden. Für die Prognosebildung beziehen sich die Lehrpersonen stärker auf die kriteriumsorientierte, namentlich wenn die Kriterien auf dem Lehrplan basieren, als auf der individualnorm-orientierten Lernprozessbeurteilung. Hingegen ziehen die Lehrpersonen die Lernprozessbeurteilung für die Einschätzung der kognitiven Fähigkeiten auch heran. Die kognitiven Fähigkeiten haben in allen Jahren einen bedeutenden Einfluss auf die Empfehlung und hier könnten auch indirekte Geschlechtereffekte auftreten. Die kognitiven Fähigkeiten von Mädchen werden bei gleichen Leistungsbeurteilungen tiefer eingeschätzt als bei den Jungen, von daher profitieren sie bei der Lehrerempfehlung in geringerem Mass von der Einschätzung der kognitiven Fähigkeiten. 
Da die Motivation nie einen statistisch signifikanten Einfluss auf die Empfehlung hat, kann man annehmen, dass zumindest über diesen Faktor keine indirekten Geschlechtseffekte auftreten. Die Annahme, dass sich über die Beurteilung von Motivation temporäres Underachievement ausgleichend in die Lehrerempfehlung einbinden lässt, kann in der vorliegenden Studie nicht schlüssig beantwortet werden. Es scheint eher so zu sein, dass sich eine erhöhte Motivation in den Leistungen manifestieren muss.

Die Lehrerempfehlung ist ein komplexes Konstrukt. Es lässt sich zwar durch Deutsch- und Mathematiklernstandesbeurteilung in grossem Mass erklären, jedoch tragen Lernstandesbeurteilung von Fächern wie Naturkunde und Französisch, sowie den Lehrerbeurteilungen von kognitiven Fähigkeiten zusätzliche Informationen bei. Die Lehrpersonen stützen ihre Empfehlung nicht rein auf dem Leistungsstand in den geprüften Fächern ab («teaching to the test»). Im dynamischen System von Freiburg kommt diesen zusätzlichen Aspekten, gerade in Fällen der bedingten Empfehlung oder von mangelnder Übereinstimmung zwischen Lehrerempfehlung und Prüfungsresultaten, eine höhere Bedeutung im Kommunikationsprozess für den definitiven Zuweisungsentscheid zu.

\section{Einschränkungen und Situierung der Studie}

Die Generalisierbarkeit der Resultate auf andere Übertrittsverfahren wird möglicherweise eingeschränkt, wenn die schulische Leistungsbeurteilung ausgeprägt ist und eine standardisierte Leistungsprüfung fehlt. Denn die Prüfung erfüllt eine wichtige Feedbackfunktion. Die Lehrpersonen haben dadurch Einsicht in die Prüfungsresultate, können diese mit ihren Empfehlungen vergleichen und haben Zugang zu einer grösseren Referenzgruppe (Watermann \& Stanat, 2004). Ebenso dämpft sie möglicherweise überhöhte Bildungsaspirationen der Eltern.

Es wäre wünschenswert gewesen, den Einfluss von sozioökonomischem Status und Muttersprache oder Kontextvariablen zu berücksichtigen. Dies war jedoch mit den vorliegenden Daten nicht möglich. Es zeigte sich an Hand der hier berichteten Analysen, dass die Auswahl von Daten einen entscheidenden Einfluss auf die Resultate hat und die Wichtigkeit von Replikationsstudien nicht unterschätzt werden darf. Die Forschungsgemeinschaft sollte gerade auch in der Bildungsforschung vermehrte Anstrengungen unternehmen, Resultate zu replizieren, um die Konsolidierung und Festigung bestehenden Wissens voranzutreiben. Speziell wenn die Erkenntnisse bildungs- und realpolitische Grundlagen sein sollen. 


\section{Anmerkungen}

1 Bezugsnormen werden kaum je in einer Reinform angewendet (Rheinberg, 2001). Hier war es der bildungspolitische Wille, sich bei der Prozessbeurteilung vor allem auf die individuell gemachten Fortschritte auszurichten. Evident bleibt, dass sich individuelle Schulleistungen grundsätzlich auf den Lehrplan beziehen.

2 Diese Praxis wurde im Verlauf der Jahre immer üblicher, deshalb nahm die Anzahl ausgeschlossener Fälle über die Jahre stetig zu.

3 Das statistische Amt des Kantons Freiburg bestätigte, dass im Schuljahr 2002/03 der Mädchenanteil in der 6 . Klasse $45.7 \%$ betrug. In dieser Zahl sind sämtliche deutschsprachigen Kinder im 6. Schuljahr erfasst. Der Unterschied von 45,7 zu 45,1\% kann auf die ausgeschlossenen Fälle zurückgeführt werden. Der Mädchenanteil desselben Jahres betrug in den französischsprachigen 6. Klassen 48,2\%. Wir danken dem statistischen Amte des Kanton Freiburg für diese Angaben. Die Analyen von Daten späterer Jahre zeigt keine Bestätigung der Tendenzen.

$4 \mathrm{DEFF}=1+($ Durchschnittliche Clustergrösse -1$) *$ ICC

5 Bei einem Vergleich von verschieden Gruppen ist es notwenig zuerst zu überprüfen, ob die latenten Konstrukte in den verschiedenen Gruppen das Gleiche bedeuten, d.h. die Faktorenstruktur nicht signifikant unterschiedlich ist (factorial validity).

6 Das Verfahren "Maximum Likelihood with robust standard errors and a mean adjusted chi-square statistic" wird auch als Yuan Bentler T2* Test Statistik bezeichnet.

7 Das gleiche Verfahren wie bei der ersten Fragestellung wurde verwendet.

\section{Literatur}

Arnold, K-H., Bos, W., Richert, P. \& Stubbe, T. C. (2007). Schullaufbahnpräferenzen am Ende der vierten Klassenstufe. In W. Bos, S. Hornberg, K.-H. Arnold, G. Faust, L. Fried, E-M. Lankes, K. Schwippert \& R. Valtin (Hrsg.), IGLU 2006. Lesekompetenzen von Grundschulkindern in Deutschland im internationalen Vergleich (S. 271-298). Münster: Waxmann.

Baeriswyl, F., Trautwein, U. \& Wandeler, C. (2008). Wie gut prognostizieren subjektive Lehrerempfehlungen und schulische Testleistungen beim Übertritt die Mathematik- und Deutschleistungen in der Sek. I? Symposiumsbeitrag anlässlich des 21. Kongresses der Deutschen Gesellschaft für Erziehungswissenschaft, 16. - 19. März 2008 in Dresden.

Baeriswyl, F., Wandeler, C., Trautwein, U. \& Oswald, K. (2006). Leistungstest, Offenheit von Bildungsgängen und obligatorische Beratung der Eltern: Reduziert das Deutschfreiburger Übergangsmodell die Effekte des sozialen Hintergrunds bei Übergangsentscheidungen? Zeitschrift für Erziehungswissenschaft, 9, 373-392.

Ball, J., Lohaus, A. \& Miebach, C. (2006). Psychische Anpassung und schulische Leistungen beim Wechsel von der Grundschule zur weiterführenden Schule. Zeitschrift für Entwicklungspsychologie und Pädagogische Psychologie, 35, 101-109.

Baumert, J. \& Schümer, G. (2001). Familiäre Lebensverhältnisse, Bildungsbeteiligung und Kompetenzerwerb. In J. Baumert, E. Klieme, M. Neubrand, M. Prenzel, U. Schiefele, W. Schneider, P. Stanat, K.-J. Tillmann \& M. Weiss (Hrsg.), PISA 2000: Basiskompetenzen von Schülerinnen und Schülern im internationalen Vergleich (S. 323-407). Opladen: Leske \& Budrich.

Baumert, J., Stanat, P. \& Watermann, R. (2006). Schulstruktur und die Entstehung differenzieller Lern- und Entwicklungsmilieus. In J. Baumert, P. Stanat \& R. Watermann (Hrsg.), Herkunftsbedingte Disparitäten im Bildungswesen: Differenzielle Bildungsprozesse und Probleme der Verteilungsgerechtigkeit (S. 95-188). Wiesbaden: VS Verlag für Sozialwissenschaften. 
Baumert, J., Trautwein, U. \& Artelt, C. (2003). Schulumwelten - institutionelle Bedingungen des Lehrens und Lernens. In Deutsches PISA-Konsortium (Hrsg.), PISA 2000. Ein differenzierter Blick auf die Länder der Bundesrepublik Deutschland (S. 261-331). Opladen: Leske \& Budrich.

Becker, M., Lüdtke, O., Trautwein, U., \& Baumert, J. (2006). Leistungszuwachs in Mathematik: Evidenz für einen Schereneffekt im mehrgliedrigen Schulsystem? Zeitschrift für Pädagogische Psychologie, 20 (4) , 233-242.

Bos, W., Lankes, E.-M., Schwippert, K., Valtin, R., Voss, A., Badel, I. \& Plassmeier, N. (2003). Lesekompetenzen deutscher Grundschülerinnen und Grundschüler am Ende der vierten Jahrgangsstufe im internationalen Vergleich. In W. Bos, E.-M. Lankes, M. Prenzel, K. Schwippert, G. Walther \& R. Valtin (Hrsg.), Erste Ergebnisse aus IGLU. Schülerleistungen am Ende der vierten Jahrgangstufe im internationalen Vergleich (S. 69-142). Münster: Waxmann.

Bos, W., Voss, A., Lankes. E.-M., Schwippert, K., Thiel, V., Valtin, R. (2004). Schullaufbahnempfehlungen von Lehrkräften für Kinder am Ende der vierten Jahrgangsstufe. In W. Bos, E.-M. Lankes, M. Prenzel, K. Schwippert, R. Valtin \& G. Walther (Hrsg.), IGLU Einige Länder der Bundesrepublik Deutschland im nationalen und internationalen Vergleich (S. 191-228). Münster: Waxmann.

Bourdieu, P. (1983). Ökonomisches Kapital, kulturelles Kapital, soziales Kapital. In R. Kreckel (Hrsg.), Soziale Ungleichheiten (S. 183-198). Göttingen: Schwartz.

Byrne, B. (2000). Structural equation model. Amos CL: Basic concepts, applications and programming (multivariate applications). New Jersey: Lawrence Erlbaum Publishers.

Ditton, H. (2007). Einleitung: Übergänge im Bildungswesen - Ergebnis rationaler Wahlen? In H. Ditton (Hrsg.), Kompetenzaufbau und Laufbahnen im Schulsystem. Ergebnisse einer Längsschnittuntersuchung an Grundschulen. Münster: Waxmann.

Ditton, H. \& Krüsken, J. (2006). Der Übergang von der Grundschule in die Sekundarstufe I. Zeitschrift für Erziehungswissenschaft, 9, 348-372.

Ditton, H., Krüsken, J. \& Schauenberg, M. (2005). Soziale Herkunft und Bildungsbeteiligung. Zeitschrift für Erziehungswissenschaft, 8, 285-304.

Elben, C. E., Lohaus, A., Ball, J. \& Klein-Hessling, J. (2003). Der Wechsel von der Grundschule zur weiterführenden Schule: Differentielle Effekte auf die psychische Anpassung. Psychologie in Erziehung und Unterricht, 50, 331-341.

Fend, H. (1981). Theorie der Schule. München: Urban \& Schwarzenbeck.

Griebel, W. (2004). Übergangsforschung aus psychologischer Sicht. In E. Schumacher (Hrsg.), Übergänge in Bildung und Ausbildung (S. 25-46). Bad Heilbrunn: Klinkhardt.

Gruehn, S. (2000). Unterricht und schulisches Lernen: Schüler als Quellen der Unterrichtsbeschreibung. Münster: Waxmann.

Ingenkamp, K. H. (1976). Schulleistung und Schulversagen als pädagogisch-diagnostische Probleme. Zur Problematik der Zensurengebung. In R. Biermann (Hrsg.), Schulische Selektion in der Diskussion (S. 79-100). Bad Heilbronn: Klinkhardt.

Ingenkamp, K. H. (1977). Die Fragwürdigkeit der Zensurengebung (7. Aufl.). Wein-

heim: Beltz.

Ingenkamp, K. H. (1985). Lehrbuch der Pädagogischen Diagnostik (Studienausgabe). Weinheim \& Basel: Beltz.

Ingenkamp, K .H. \& Lissmann, U. (2005). Lehrbuch der Pädagogischen Diagnostik. Weinheim: Beltz.

Jürgens, E. (1989). Lehrer empfehlen Eltern entscheiden. Zur Übergangsproblematik der Orientierungsstufe vor dem Hintergrund der Bewährung empfohlener und nichtempfohlener Schüler im weiterführenden Schulsystem. Die Deutsche Schule 81, 3, 388-400.

Klauer, K. J. (1987). Fördernde Notengebung durch unter drei Bezugsnormen. In R. Olechowsky \& E. Persy (Hrsg.), Fördernde Leistungsbeurteilung (S. 180-206). Wien: Jugend und Volk. 
Krapp, A. (1979). Prognose und Entscheidung. Zur theoretischen Begründung und Differenzierung der pädagogisch-psychologischen Prognose. Weinheim: Beltz.

Maaz, K., Neumann, M. \& Trautwein, U. (in Druck). Schulsysteme im deutschsprachigen Raum. In W. Sacher, L. Haag, T. Bohl, G. Lang-Wojtasik \& S. Blömeke (Hrsg.), Handbuch Schule: Theorie, Geschichte, Organisation, Entwicklung, Evaluation. Bad Heilbrunn: Klinkhardt.

Mischo, C. \& Rheinberg, F. (1995). Erziehungsziele von Lehrern und individuelle Bezugsnormen der Leistungsbewertung. Zeitschrift für Pädagogische Psychologie, 9, 139-152.

Muthén, L. K., \& Muthén, B. O. (1998-2006). Mplus user's guide (4th ed.). Los Angeles, CA: Muthén \& Muthén.

Muthén, L. K., \& Muthén, B. O. (1999). Intraclass correlations: size of design effect. Notes on Mplus website, letzter Zugriff 27.11.2008 from www.statmodel.com

Muthén, L. K., \& Muthén, B. O. (2005). Chi-square difference testing using the S-B scaled chi-square. Notes on Mplus website, letzter Zugriff 27.11.2008 from www.statmodel.com

Neuenschwander, M. P. (2007). Bedingungen und Anpassungsprozesse bei erwartungswidrigen Bildungsverläufen. In T. Eckert, (Hrsg.), Übergänge im Bildungswesen (S. 83-104). Münster: Waxmann.

Neuenschwander, M. P., Balmer, T., Gasser, A. Golz, S., Hirt, U. et al. (2005). Schule und Familie - was sie zum Schulerfolg beitragen. Bern: Haupt.

Neumann, M., Schnyder, I., Trautwein, U., Niggli, A., Lüdtke, O. \& Cathomas, R. (2007). Schulformen als differenzielle Lernmilieus: Institutionelle und kompositionelle Effekte auf die Leistungsentwicklung im Fach Französisch. Zeitschrift für Erziehungswissenschaft, 10, 399-420.

Ophuysen van, St. (2006). Zur Problematik der Schulformempfehlungen nach der Grundschulzeit und ihre prognostische Qualität. In W. Bos, H. G. Holtappels, H. Pfeiffer, H.G. Rolff, R. Schulz-Zander (Hrsg.), Jahrbuch der Schulentwicklung. Daten Beispiele und Perspektiven (S. 49 - 79). Weinheim und München. Juventa.

Raudenbush, S.W. \& Bryk, A. S. (2002). Hierarchical linear models (2nd ed.). Thousand Oaks, CA: Sage.

Rheinberg, F. (1982). Bezugsnorm-Orientierung von Lehramtsanwärtern im Verlauf ihrer praktischen Ausbildung. In F. Rheinberg (Hrsg.), Jahrbuch für Empirische Erziehungswissenschaft 1982 (S 235-248). Düsseldorf: Schwann.

Rheinberg, F. (2001). Bezugsnormen und schulische Leistungsbeurteilung. In F. Weinert (Hrsg.), Leistungsmessungen in der Schule (S. 59-71). Weinheim und Basel: Beltz.

Rheinberg, F. (2006). Bezugsnorm-Orientierung. In D. H. Rost (Hrsg.), Handwörterbuch Pädagogische Psychologie (3. Aufl., S. 55-62). Weinheim: Beltz.

Rheinberg, F. \& Krug, S. (1999). Motivationsförderung im Schulalltag (2. Aufl.). Göttingen: Hogrefe.

Sauer, J. \& Gamsjäger, E. (1996). Ist Schulerfolg vorhersagbar? Göttingen: Hogrefe.

Schwegler, M. (1989). Fördern und Fordern des Schülers. Unveröffentlichte Lizentiatsarbeit, Universität Freiburg/Schweiz, Philosophische Fakultät.

Stahl, N. (2007). Schülerwahrnehmung und -beurteilung durch Lehrkräfte. In H. Ditton, (Hrsg.), Kompetenzaufbau und Laufbahnen im Schulsystem. Ergebnisse einer Längsschnittuntersuchung an Grundschulen (S. 171-198). Münster: Waxmann.

Stubbe, T. C. \& Bos, W. (2008). Schullaufbahnempfehlungen von Lehrkräften und Schullaufbahnempfehlungen von Eltern am Ende der vierten Jahrgangsstufe. Empirische Pädagogik, 22 (1), 49-63.

Thiel, O. (2006). Modellierung der Bildungsgangempfehlung in Berlin. Inauguraldissertation. Berlin: Humboldt-Universität. letzter Zugriff 27.11.2008 http://deposit.ddb.de/cgibin $/$ dokuserv?idn=979175313

Trautwein U., Baeriswyl, F., Lüdcke, O. \& Wandeler, C. (in Druck). Die Öffnung des Schulsystems: Fakt oder Fiktion? Empirische Befunde zum Zusammenhang von Grundschulübertritt und Übergang in die gymnasiale Oberstufe im Kanton Freiburg. 
Valtin, R. \& Wagner, C. (2004). Der Übergang in die Sekundarstufe I: Psychische Kosten der externen Leistungsdifferenzierung. Psychologie in Erziehung und Unterricht, 51, 52-68.

Vögeli-Mantovani, U. (1999). Mehr fördern, weniger auslesen. Zur Entwicklung der schulischen Beurteilung in der Schweiz. Aarau: Schweizerische Koordinationsstelle für Bildungsforschung.

Watermann, R. \& Stanat, P. (2004). Schulrückmeldungen in PISA 2000: Sozialnorm- und kriteriumsorientierte Rückmeldeverfahren. Empirische Pädagogik, 18 (1), 40-61.

Ziegenspeck, J. (1973). Zensur und Zeugnis in der Schule. Hannover: Schrödel.

Schlagworte: Übertrittsverfahren, Schul- und Klassenübergang, Lehrerempfehlungen, schulische Leistungsbeurteilung, Bezugsnormen, Leistungstest, Reliabilität der Lehrempfehlungen

\section{Analyses des déterminations et constances des recommandations des enseignants lors de la transition sur sept ans}

\section{Résumé}

Les transitions sont des caractéristiques du système scolaire de l'Europe centrale et elles représentent des étapes importantes pour l'enfant. Dans les systèmes scolaires divisés en filières, l'entrée au secondaire I est particulièrement importante: l'attribution à une filière est implicitement reliée à l'orientation professionnelle. Pour l'orientation scolaire, la prise en compte de la recommandation de l'enseignant est un critère courant. Cet article examine, sur une durée de sept ans, si ce qui détermine les recommandations des enseignants varie au cours des années et si un modèle stable peut être dégagé des recommandations des professeurs. Les données à propos de la transition entre l'école primaire et le secondaire I ont été recueillies auprès de 5332 élèves germanophones du canton de Fribourg (Suisse). Sur la base d'un découpage des données par classe, le même modèle a été appliqué pour les sept années. La modélisation des recommandations des enseignants fait apparaître que, d'une année à l'autre, les différences statistiques ne sont pas significatives, même si, dans la pondération des variables, des tendances partiellement différentes s'observent. Il ressort que les enseignants prennent en compte les performances plutôt que les processus. De plus, les évaluations du niveau atteint en mathématiques et en allemand ainsi que les capacités cognitives représentent les facteurs prédictifs les plus manifestes.

Mots clés: Procédures de passage, transitions scolaire et d'une classe, recommandations des eneignants, jugement de performance scolaire, tests de performance, fiabilité du jugement 


\section{Le proposte per il passaggio di settore scolastico - casuali o attendibili? Analisi delle determinanti e costanza delle proposte di passaggio degli insegnanti sull'arco di sette anni}

\section{Riassunto}

Le transizioni sono un aspetto importante del sistema scolastico dell'Europa centrale e sono delle fasi importanti per il bambino. Nei sistemi scolastici strutturati in percorsi con esigenze differenziate, l'entrata nel ciclo di orientamento è particolarmente importante, perché implicitamente anche l'attribuzione delle possibilità di formazione è legata alla ripartizione in sezioni scolastiche. Per la ripartizione, la raccomandazione dell'insegnante è un criterio molto diffuso. Questo articolo esamina se le determinanti della raccomandazione degli insegnanti variano sull'arco di sette anni consecutivi e se il modello della raccomandazione dei docenti sia stabile. Sono stati raccolti i dati della procedura di transizione tra scuola primaria e scuola media di 5332 allievi germanofoni del Canton Friborgo (Svizzera), utilizzando lo stesso modello applicato a sette anni differenti. La modellizzazione delle raccomandazioni degli insegnanti non ha messo in rilievo delle differenze statistiche significative. Gli insegnanti giudicano più in base alle prestazioni che ai processi. Le prestazioni di matematica e di tedesco, così come le capacità cognitive sono infine i fattori predittivi più importanti.

Parole chiave: processi di transizione, passaggio scolastico e passaggio di classe, raccomandazione del professore, giudizio di perfomanza scolastica, test di perfomanza.

\section{The school transition recommendation - Analysis of the determinants and stability of teachers' transition recommendation over seven years}

\section{Summary}

Transitions are a central feature of the Central European school system and are important stages for a child. For school systems with different achievement-based secondary school tracks, the entry into the secondary school is particularly important, because the assignment to one of the tracks is implicitly also connected to the allocation of educational opportunities. For the tracking, the teacher recommendation is a widespread criterion. This paper investigates whether the determinants of teachers recommendations from seven consecutive years differ and if a stable model of the teacher recommendation can be found.

The data stem from 5,332 pupils in the transition process from primary to secondary school of the German-speaking schools in the canton of Freiburg (Switzerland). Taking into account the nested data on the class level the same model is specified for seven consecutive years. The model of the teachers' recommenda- 
tions does not differ statistically significant. Teachers consider the performance evaluation more than the process evaluations. The strongest predictors are the Mathematics and German grades, as well as the estimated cognitive skills.

Key words: transition, school and class transition, teacher recommendations, academic performance assessment, performance test, reliability 